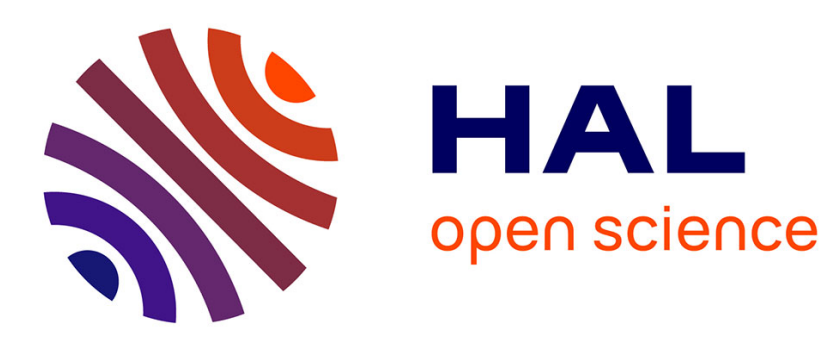

\title{
The micromechanical nature of stresses in triphasic granular media with interfaces
}

Jérôme Duriez, M. Eghbalian, R. Wan, F. Darve

\section{To cite this version:}

Jérôme Duriez, M. Eghbalian, R. Wan, F. Darve. The micromechanical nature of stresses in triphasic granular media with interfaces. Journal of the Mechanics and Physics of Solids, 2017, 99, pp.495 511. 10.1016/j.jmps.2016.10.011 . hal-01877084

\section{HAL Id: hal-01877084 \\ https://hal.science/hal-01877084}

Submitted on 22 Oct 2018

HAL is a multi-disciplinary open access archive for the deposit and dissemination of scientific research documents, whether they are published or not. The documents may come from teaching and research institutions in France or abroad, or from public or private research centers.
L'archive ouverte pluridisciplinaire HAL, est destinée au dépôt et à la diffusion de documents scientifiques de niveau recherche, publiés ou non, émanant des établissements d'enseignement et de recherche français ou étrangers, des laboratoires publics ou privés. 


\title{
The micromechanical nature of stresses in triphasic granular media with interfaces
}

\author{
J. Duriez ${ }^{\mathrm{a}}$, M. Eghbalian ${ }^{\mathrm{a}}$, R. Wan ${ }^{\mathrm{a}}$, F. Darve ${ }^{\mathrm{b}}$ \\ ${ }^{a}$ University of Calgary, Calgary, AB, T2N 1N4, Canada \\ ${ }^{b}$ Univ. Grenoble Alpes, 3SR, F-38000 Grenoble, France \\ CNRS, 3SR, F-38000 Grenoble, France
}

\begin{abstract}
The total stress tensor as the average stress within a triphasic granular medium is formally derived from micromechanics where internal forces associated with the solid phase, the two immiscible fluid phases and the associated three interfaces are explicitly accounted for. It is demonstrated that for rigid solid particles, the contributions of all local solid-fluid surface tensions to the total stress are eventually zero. The present work gives the total stress expression as a function of a solid-phase specific stress tensor and a fluid mixture stress contribution that is related to the material's microstructure. A generally non-spherical fluid mixture stress is obtained in contrast to an averaged hydrostatic fluid pressure usually associated with standard thermodynamics. The tensorial nature of this fluid mixture stress contribution is highlighted through numerical experiments pertaining to an idealized granular material in the pendular regime at low wetting saturations. Numerical simulations providing full access to microstructural information are conducted using the Discrete Element Method (DEM), which describes internal forces using resultant forces that clearly deviate from the distributed nature of internal forces in triphasic granular media, e.g., fluid pressures. Nevertheless, this micro-scale representation is demonstrated to be indeed valid for macro-scale stress description in the pendular regime.

Keywords: granular material, microstructure, stress, unsaturated conditions, interfaces
\end{abstract}

Preprint submitted to Elsevier

October 22, 2018 


\section{Introduction}

Granular materials have many distinctive features, revealing among others, a dual nature: they can behave as either a fluid or a bulk solid as observed in an hourglass. In the bulk solid state, they possess mechanical strength and bearing capacity so that they are able to sustain foundation loads such as in civil engineering. However, this mechanical strength can drastically change in the presence of a mixture of fluids within the pore space due to the multiphasic condition that sets in, implicating various interactions between the individual solid and fluids phases. In the real world, this phenomenon is clearly evidenced by the change in consistency of sand in a beach as the water content gradually increases towards the shoreline.

As will become more evident later throughout this paper, challenges in the mechanical description of such a multiphasic porous material arise from the variety of stress variables that emerge. On the one hand, the equilibrium under external loads applied on the boundaries of a triphasic granular system involves an overall macroscopic stress, the so-called total stress. Probing into the microscopic scale on the other hand, shows that distinct stresses also exist in the fluids and the solid phase, with for instance distinct individual fluid pressures since the fluid phases are separated by singular surfaces showing interfacial surface tension.

A simplified interpretation of the behaviour through one single stress variable would be of great practical interest, which conceptually led to the Principle of Effective Stress (Terzaghi, 1936; Bishop and Blight, 1963; Nuth and Laloui, 2008). This concept delivers a very strong statement as it signifies that the mechanical response (strains) of a material is solely controlled by a so-called effective stress related to the solid phase, irrespective of the magnitudes of any of the stresses in the various phases. In other words, when comparing a dry (airsaturated) porous material with when it is invaded with various fluid phases, the Principle of Effective Stress implies that the same mechanical behaviour is to be expected as long as the effective stress loading path under multiphasic 
conditions coincides with the total stress path in the dry state.

The effective stress concept has been shown to be valid when the pore space is saturated by one single fluid, with the effective stress $\sigma^{\prime}$ being expressed under such conditions in terms of the total stresses $\Sigma$, the fluid pressure $u$, the solid matrix compressibility $K_{s}$ and the drained bulk compressibility (of the skeleton) $K_{h}$ (Biot, 1941):

$$
\boldsymbol{\sigma}^{\prime}=\boldsymbol{\Sigma}-u\left(1-\frac{K_{h}}{K_{s}}\right) \boldsymbol{\delta} \approx \boldsymbol{\Sigma}-u \boldsymbol{\delta}
$$

where $\boldsymbol{\delta}$ is the identity tensor. Interestingly, the approximated version of Eq. (11), as proposed by Terzaghi (1936), is the most venerable equation used in soil mechanics where soil particles are much stiffer than the skeleton as a whole.

By construction, Eq. (10) is restricted to the biphasic case, which led Bishop $(1959 ; 1963)$ to address the more commonly encountered triphasic condition, proposing a variant of Terzaghi's effective stress expression, i.e.

$$
\boldsymbol{\sigma}^{\prime}=\boldsymbol{\Sigma}-u_{n}(1-\chi) \boldsymbol{\delta}-u_{w} \chi \boldsymbol{\delta}
$$

with $\chi \in[0 ; 1]$ the so-called Bishop parameter, and $u_{n}, u_{w}$ the pressures of the non-wetting $(n)$ and wetting $(w)$ immiscible fluid phases, respectively. Water and air in so-called unsaturated soils, or water and oil in petroleum reservoirs are typical examples of wetting and non-wetting fluids - with the wetting fluid type being dependent on the host soil or rock for oil-water mixtures.

Unfortunately, the application of Eq. (2) to experimental observations on triphasic media faced major difficulties, as reviewed by Nuth and Laloui (2008). In particular, no stress variable was found by Nuth and Laloui (2008) that would relate to strain in a consistent manner between unsaturated and dry conditions, thus challenging the principle of effective stress. The same conclusion has been reached by Wan et al. (2015) based on the numerical modelling of dry and unsaturated granular media. On the other hand, considering a solid-phase related stress variable, Khalili et al. (2004): Lu and Likos (2006): Nuth and Laloui (2008) obtained a unique stress limit criterion from experimental data at failure in both triphasic and biphasic conditions, which has also been obtained numerically by Hicher and Chang (2007); Scholtès et al. (2009); Wan et al. 
(2015). In the end, a consensus is that some aspects of the mechanical behaviour in triphasic conditions, such as failure description, can be inferred from biphasic conditions using a single effective stress variable, whereas a full stress-strain description requires including additionnal stress variables, e.g. the capillary pressure $u_{r}=u_{n}-u_{m}$, in constitutive models (Nuth and Laloui. 2008: Alonso et al., 2010). Basically, this means that the Principle of Effective Stress as stated previously does not hold in triphasic conditions. Nevertheless, it appears that the effective stress denomination is often still associated to the solid-phase related variable mentionned in the above (Nuth and Laloui, 2008: Borja and Koliji, 2009; (Alonso et al., 2010). Connected with the possible breakdown of the Principle of Effective Stress is the "two stress variables approach" that does not rely on any effective stress (Fredlund, 2006; Alonso et al., 1990), as well as the suction hardening concept according to which the capillary pressure enters the hardening parameters (Boria and Koliiil, 2009), leading to discrepancies between biphasic and triphasic conditions.

The search for a single effective stress variable in the realm of geomechanics may be formally compared to the search for a back stress variable, as coined by Nowick and Machlin (1947), in Solid Mechanics. Tensorial back stress variables enter elasto-viscoplastic models as the difference between the total stress tensor and a dissipative stress variable which governs the material behaviour (Prager), 1956; Lemaitre and Chaboche. 1990: Frederick and Armstrong. 2007: Henann and Anand,2009). Evolving yield surface centers in kinematic hardening models are typical back stress examples.

Modelling triphasic porous media according to the Principle of Effective Stress would also require subtracting from the total stress a stress contribution accounting for internal forces due to the fluid mixture, leaving the solid-phase related effective stress to govern material deformation and failure. The fluid mixture stress contribution is denoted as suction stress in the general case ( $\mathrm{Lu}$ and Likos, 2006; Nikooee et al., 2013) and reduces to the so-called capillary stresses (Hicher and Chang, 2007; Scholtès et al., 2009; Wan et al., 2015) for granular media with non-viscous fluids. Indeed, the pore fluid mixture is exclu- 
sively governed by capillarity in such media, with other physico-chemical forces and adsorbed liquid layers being negligible. The present manuscript focuses on capillary stresses only, hoping that a correct capillary stress determination as proposed herein will subsequently bring some insights into a more general effective stress discussion. At the moment, the following results have been obtained for the nature of capillary stresses.

In line with Bishop's (1959) expression given in Eq. (2), capillary stresses are often expressed in terms of the fluid pressures only (e.g., Zienkiewicz et al., 1999; Nuth and Laloui, 2008; Borja and Koliji, 2009; Lu et al., 2010). However, triphasic conditions imply singular discontinuity surfaces as fluid-fluid interfaces which are absent in the biphasic case. This motivated other approaches to include interfacial surface tension forces in the stress analysis. Among those, Fredlund (2006); Madeo et al. (2013); Duriez and Wan (2016b) account for the fluid-fluid interface only, while Grav et al. (2009); Nikooee et al. (2013) include solid-fluid interfaces as well. The lack of consensus may arise from the fact that capillarity is best evidenced and understood for fluid-fluid interfaces e.g., in capillary tubes, leading even to controversies about solid-fluid interfaces in the fluid mechanics community (Lunati, 2007; Shikhmurzaev, 2008). Nevertheless, solid-fluid surface tensions, being described as early as in Young's equation (1805), are evidenced by the contraction of very small metal particles (e.g. Vermaak et al., 1968; Jiang et al., 2001) or may let sand adopt a hydrophobic behaviour (Vitz, 1990).

A first objective of the present work is to clarify under which conditions the solid-fluid interfaces contribute or not to the stress of triphasic granular media. In fact, it will be rigorously demonstrated that the stress contribution of solid-fluid surface tension is zero on average for negligible solid-solid surfaces, an important result absent from previous works bearing similarities with Section 2 such as Chateau et al. (2002); Dormieux et al. (2006a); Gray et al. (2009).

Also, most approaches represent the capillary stresses as a scalar or a spherical, i.e. isotropic, tensor (Hassanizadeh and Grav, 1990; Nuth and Laloui, 2008; Borja and Koliji, 2009; Gray et al., 2009; Lu et al., 2010; Nikooee et al., 2013). 
This feature arises in these works from the usual consideration of mixture theory and thermodynamics framework, often adopting a restrictive scalar or spherical description of stresses and strains. Indeed, other micromechanical approaches suggest that capillary stresses show a generic tensorial nature (Chateau et al., 2002; Hicher and Chang. 2007: Scholtès et al.. 2009: Wan et al.l. 2015: Duriez and Wan, 2016b; Li et al., 2016). It is tempting to consider micromechanics to bring more pertinent insights to the mechanical behaviour, such as done by Sibille et al. (2015), because granular materials show a clear microstructure with also a fluid 'fabric' in triphasic conditions (Manahiloh and Muhunthan, 2012).

A second objective of the manuscript is thus to clearly establish and illustrate how the microstructure enters into the stress state of triphasic granular media, leading to deviatoric capillary stresses as shown in Section 4 . It is noteworthy that Madeo et al. (2013) evidenced the need of a second gradient theory to describe the mechanical behaviour of unsaturated porous media. However, the scope of the present paper is restricted to the stress tensor and the first displacement gradient being work-conjugate.

The present paper begins with the micromechanical derivation of stresses in a triphasic granular medium in Section 2, isolating the sought after capillary stresses. A clear microstructure dependency is proposed for the capillary stresses, and because experimental microstructure measurements in triphasic granular media, e.g from X-ray computed tomography (Culligan et al., 2004; Bruchon et al., 2013), are not yet precise enough to be used in conjunction with the analytical developments presented herein, Section 3 recalls a numerical model of ideal triphasic granular media at low wetting saturation that provides full access to pertinent microstructural information, including interfaces (Duriez and Wan, 2016a). Based on the Discrete Element Method (Cundall and Strack, 1979), the model describes by design the micro-scale stress field from resultant vector forces, that are distinct in nature from distributed fluid pressure or surface tension internal forces. Chateau et al. (2002); Duriez and Wan (2016b) nevertheless suggested that such DEM models are still able to describe 
stresses in triphasic granular media, but in the absence of any formal analytical demonstration.

Hence, a third objective of the paper is to formally provide such a demonstration as touched upon in Section 3.2. Finally, Section 4 explores in details important characteristics and properties of the analytically derived capillary stresses with the aid of DEM.

The notation adopted in this paper is such that a superimposed arrow designates first order tensors (vectors) such as $\vec{x}, \vec{\nu}$, and bold symbols are employed for second order tensors like $\boldsymbol{\delta}, \boldsymbol{\Sigma}$. Also, the geomechanics sign convention is used, i.e. $\boldsymbol{\sigma} \vec{n}$ corresponds to an applied loading on a given body when the normal vector $\vec{n}$ points inwards to the boundary, leading to a positive sign for compressive stresses $\sigma$.

\section{Stress homogenization of triphasic granular media with interfaces}

The representative elementary volume (REV) $V$ of a triphasic granular material features, at the micro-scale, different volume phases $V_{\alpha}, \alpha \in\{n ; s ; w\}$ for the solid $(s)$ and fluids $(n, w)$ phases, as well as interface surfaces $S_{\alpha \beta}, \alpha$ and

$\beta \neq \alpha \in\{n ; s ; w\}$, and contact lines $\Gamma$ where all three phases meet. Extending Duriez and Wan (2016b), surface tension is here described along all three interfaces, and we first underscore the corresponding consequences that ensue for the stress description at the micro-scale.

\subsection{Micro-scale stress description along interfaces and contact lines}

An interface $S_{\alpha \beta}$ considered here as a distinct medium is subject to internal surface tension forces proportional to the surfacic energy $\gamma_{\alpha \beta}$. The following stress-like tensor $\boldsymbol{\pi}_{\boldsymbol{\alpha} \boldsymbol{\beta}}$ describes these internal surface tension forces that are tensile in nature (Chateau et al., 2002; Dormieux et al., 2006b; Gray et al., 2009; Duriez and Wan, 2016b):

$$
\boldsymbol{\pi}_{\boldsymbol{\alpha} \boldsymbol{\beta}}=\gamma_{\alpha \beta}(\vec{n} \otimes \vec{n}-\boldsymbol{\delta})
$$


Following the geomechanics sign convention, Eq. (3) basically expresses the surface stress-like tensor $\boldsymbol{\pi}_{\boldsymbol{\alpha} \boldsymbol{\beta}}$ in terms of $\gamma_{\alpha \beta}$ and the projection tensor onto $S_{\alpha \beta}, \delta_{\boldsymbol{\alpha} \boldsymbol{\beta}}=(\boldsymbol{\delta}-\vec{n} \otimes \vec{n})$, with $\boldsymbol{\delta}$ the identity tensor, and $\vec{n}$ the normal to $S_{\alpha \beta}$.

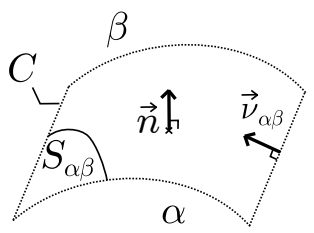

Figure 1: Interface $S_{\alpha \beta}$ with $\vec{n}$ the normal and $\vec{\nu}_{\alpha \beta}$ the inwards conormal

The interface material points experience along the contour $C$ the surface tension forces described by $\boldsymbol{\pi}_{\boldsymbol{\alpha} \boldsymbol{\beta}}$, as well as internal forces within $\alpha$ (described by Cauchy stress $\sigma_{\alpha}$ ), and internal forces within $\beta$ (described by $\boldsymbol{\sigma}_{\boldsymbol{\beta}}$ ). Hence, the equilibrium of forces at the interface material points leads to:

$$
\int_{S_{\alpha \beta}}\left(\sigma_{\alpha}-\sigma_{\beta}\right) \vec{n} d S-\int_{C} \gamma_{\alpha \beta} \vec{\nu}_{\alpha \beta} d l=\overrightarrow{0}
$$

with $\vec{n}$ pointing from $\alpha$ to $\beta$ and $\vec{\nu}_{\alpha \beta}$ the inward conormal tangent to $S_{\alpha \beta}$ and normal to $C$, and $d l>0$ the arc length (Fig. 1). Next, the contour integral in Eq. (44) is classically rewritten using the following equality (see e.q.. Gurtin and Murdoch, 1975; Wan et al., 2015):

$$
\int_{C} \vec{\nu}_{\alpha \beta} d l=\int_{S_{\alpha \beta}} \operatorname{div}_{\mathrm{S}}(\vec{n}) \vec{n} d S
$$

with $\operatorname{div}_{\mathrm{S}}()$ the surface divergence operator as defined by Gurtin and Murdoch (1975): roughly speaking, $\operatorname{div}_{S}()$ refers to the projection of the classical differential operator $\nabla \cdot=\operatorname{div}()$ onto the tangent plane of the surface $S$ of interest. Eq. (5) finally gives the normal stress discontinuity across $S_{\alpha \beta}$ due to capillarity; in case $S_{\alpha \beta}$ is curved:

$$
\left(\boldsymbol{\sigma}_{\boldsymbol{\alpha}}-\boldsymbol{\sigma}_{\boldsymbol{\beta}}\right) \vec{n}=\gamma_{\alpha \beta} \operatorname{div}_{\mathrm{S}}(\vec{n}) \vec{n}
$$

Considering the fluid-fluid interface $S_{n w}, \sigma_{\alpha}=u_{\alpha} \delta, \alpha \in\{n ; w\}$ with $u_{\alpha}$ the fluid pressures, together with Eq. (6) give the Laplace-Young equation:

$$
u_{c}=u_{n}-u_{w}=\gamma_{n w} \operatorname{div}_{\mathrm{S}}(\vec{n})
$$


with $\vec{n}$ towards the wetting fluid. The capillary pressure $u_{c}$ can be identified as the matric suction in the present case of granular media.

Considering the solid-fluid interface $S_{s \beta}$, Eq. (6) serves as a boundary condition for the solid stresses that accounts for solid-fluid surface tension (Chateau et al., 2002; Dormieux et al., 2006a; Lunati, 2007; Gray et al., 2009), with $\vec{n}$ being the outward solid normal:

$$
\sigma_{s} \vec{n}=\left(u_{\beta}+\gamma_{s \beta} \operatorname{div}_{\mathrm{S}} \vec{n}\right) \vec{n}
$$

A different boundary condition for the solid stresses holds along the contact line $\Gamma$ where the solid phase meets the two fluid phases (Fig. 21). Here, equilib-
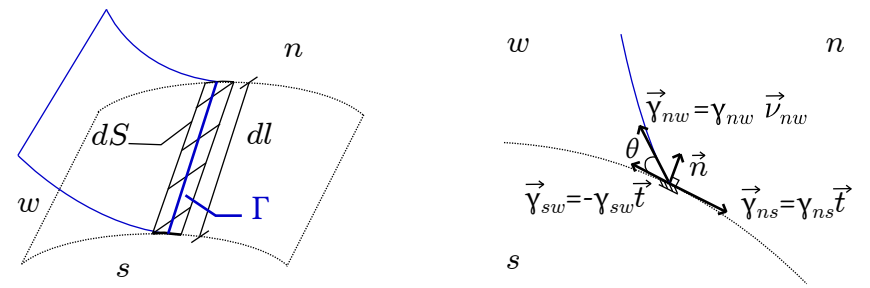

Figure 2: Contact line $\Gamma: 3 \mathrm{D}$ (left) and 2D (right) views. With respect to Eq. 9), $d l$ is a portion of $\Gamma$, and $d S$ is an elemental surface area constructed from $d l$ and an infinitesimally small length (magnified in the Figure) along $\vec{t}$

rium consideration for a peripheral solid facet $d S$ located along $\Gamma$ and sustaining solid internal forces and three surface tension forces leads to:

$$
\boldsymbol{\sigma}_{\boldsymbol{s}} \vec{n} d S+\left(\vec{\gamma}_{n w}+\vec{\gamma}_{n s}+\vec{\gamma}_{s w}\right) d l=\overrightarrow{0} \Leftrightarrow \boldsymbol{\sigma}_{\boldsymbol{s}} \vec{n} d S=\left(-\vec{\gamma}_{n w}+\left(\gamma_{s w}-\gamma_{n s}\right) \vec{t}\right) d l(9)
$$

where $\vec{n}$ is the outward solid normal and $\vec{t}$ a tangent vector. In connection with Young's equation (1805) given as

$$
\gamma_{n w} \cos \theta=\gamma_{n s}-\gamma_{s w}
$$

with $\theta$ being the contact or wetting angle (Fig. 2), a local zero tangential solid stress along $\Gamma$ can be deduced from Eq. (9):

$$
\left(\sigma_{s} \vec{n}\right) \cdot \vec{t}=\gamma_{n w} \cos \theta+\gamma_{s w}-\gamma_{n s}=0
$$

Now that the micro-scale stress description has been recalled, the macroscale stress tensor is next derived. 


\subsection{Macro-scale stresses of triphasic granular media}

The total stress $\boldsymbol{\Sigma}$ for the REV is volume-averaged using the microscopic stress tensors within all three volume phases and three interfaces (Chateau et al., 2002; Dormieux et al., 2006a; Gray et al., 2009). Classically, no stress tensor or other mechanical properties is considered for the contact line itself (Hassanizadeh and Grav, 1990; Grav et al., 2009; Nikooee et al., 2013):

$$
\boldsymbol{\Sigma}=\frac{1}{V}\left[\sum_{\alpha=n, s, w} \int_{V_{\alpha}} \boldsymbol{\sigma}_{\boldsymbol{\alpha}} d V+\sum_{\alpha, \beta=n, s, w} \int_{S_{\alpha \beta}} \boldsymbol{\pi}_{\boldsymbol{\alpha} \boldsymbol{\beta}} d S\right]
$$

The fluid stresses are the isotropic pressures $u_{n} \boldsymbol{\delta}, u_{w} \boldsymbol{\delta}$. Considering thermodynamic equilibrium, the fluid pressures are assumed to be uniform, leading to a uniform capillary pressure, whatever the saturation regime and the connected or disconnected feature of the fluid phases.

Regarding the solid phase, the latter is made of distinct particles $p, V_{s}=$ $\bigcup V_{p}$, and the volume integral for each particle is classically rewritten as a surface integral from the divergence theorem, considering equilibrium of particles without body forces:

$$
\int_{V_{p}} \boldsymbol{\sigma}_{\boldsymbol{s}} d V=\int_{S_{p}}\left(\boldsymbol{\sigma}_{\boldsymbol{s}} \vec{n}\right) \otimes \vec{x} d S
$$

with $\vec{n}$ the outward normal and the positions $\vec{x}$ being defined from an adequate centroid for each particle thanks to particles equilibrium.

Paying attention to the microstructure of triphasic granular media, the particle surfaces $S_{p}$ show solid-fluid interfaces $S_{p, s \alpha}, \alpha \in\{n ; w\}$, contact lines $\Gamma_{p}$, and contact surfaces $S_{p, s s}$. Eq. (8), resp. (9), applies along $S_{p, s \alpha}$, resp. $\Gamma_{p}$. Considering quasi-rigid particles, the contact surfaces $S_{p, s s}$ are negligible and thus,

$$
\int_{S_{p, s s}}\left(\boldsymbol{\sigma}_{\boldsymbol{s}} \vec{n}\right) \otimes \vec{x} d S=-\sum_{c_{p}} \vec{f}^{c} \otimes \vec{x}^{c}
$$

with $\vec{f}^{c}$ being the contact forces, as sustained by $p$, due do the different contacts $\left\{c_{p}\right\}$. 
Accounting for the above, algebraic manipulations of Eq. (12) lead to the following expression for the total stresses:

$$
\begin{aligned}
\boldsymbol{\Sigma}-u_{n} \boldsymbol{\delta}=\frac{1}{V} \quad & {\left[\sum_{c} \vec{f}^{c} \otimes \vec{l}-u_{c}\left(V_{w} \boldsymbol{\delta}+\int_{S_{s w}} \vec{n} \otimes \vec{x} d S\right)\right.} \\
& -\gamma_{n w}\left(\int_{S_{n w}}(\boldsymbol{\delta}-\vec{n} \otimes \vec{n}) d S+\int_{\Gamma} \vec{\nu}_{n w} \otimes \vec{x} d l\right) \\
& \left.+\sum_{\alpha=n ; w} \gamma_{s \alpha}\left(\int_{S_{s \alpha}}\left(\operatorname{div}_{S} \vec{n} \vec{n} \otimes \vec{x}+\vec{n} \otimes \vec{n}-\boldsymbol{\delta}\right) d S-\int_{\Gamma} \vec{\nu}_{s \alpha} \otimes \vec{x} d l\right)\right]
\end{aligned}
$$

The above stress expression together with the physical interpretation of each term will be amply discussed in the following sections.

\subsection{Solid-fluid surface tension zero stress contribution}

We herein focus on the last stress contributional term on the r.h.s of Eq. (15), arising from solid-fluid surface tensions $\gamma_{s \alpha}$. First, we observe that such stress contributions are due directly to the internal forces within the interfaces $S_{s \alpha}$ by integrating surface tension force field $\boldsymbol{\pi}_{\boldsymbol{s} \boldsymbol{\alpha}}$ over the membrane surface. Other $\gamma_{s \alpha}$-stress contributions come from the stress application by $S_{s \alpha}$ onto the solid phase, through the two boundary conditions for the solid stresses, i.e. Eq. (8) along the surfaces $S_{s \alpha}$ excluding the contact lines, and Eq. (9) along the contact

lines. However, these $\gamma_{s \alpha}$-stress contributions cancel out for each interface since the following equality holds for any surface $S$ of contour $C=\Gamma$, conormal $\vec{\nu}$, and normal $\vec{n}$ (Fig. 11):

$$
\int_{S}\left(\operatorname{div}_{\mathrm{S}}(\vec{n}) \vec{n} \otimes \vec{x}+\vec{n} \otimes \vec{n}-\boldsymbol{\delta}\right) d S=\int_{\Gamma} \vec{\nu} \otimes \vec{x} d l
$$

Eq. (16) is formally demonstrated using Stokes theorem in Appendix A. In fact, this important result is a generalization of the following equation proposed by Rosenkilde (1967) that is restricted to closed surfaces $S_{c}$ :

$$
\int_{S_{c}} \operatorname{div}_{\mathrm{S}}(\vec{n}) \vec{n} \otimes \vec{x} d S=\int_{S_{c}}(\boldsymbol{\delta}-\vec{n} \otimes \vec{n}) d S
$$

Obviously, Eq. (16) reduces to (17) for closed surfaces showing no contour $\Gamma$.

The conclusion of a zero average stress contribution from solid-fluid surface tensions is valid for any porous media including one or two immiscible fluids and showing negligible solid-solid surfaces $S_{s s}$ such that the solid-fluid 
surfaces $S_{s \alpha}$ are always bound by contact lines $\Gamma$. In this sense, the present result obtained from quasi-static micromechanics is consistent with the thermodynamical approach followed by Nikooee et al. (2013). Indeed, Nikooee et al. (2013) also showed, from alternative free energy considerations, that solid-fluid surface tensions would affect the behaviour only for deformable solid particles. It should be noted that Chateau et al. (2002) previously mentioned this zero stress contribution from solid-fluid surface tensions without a clear justification. Also, it is worth mentioning that the herein proposed Eq. (16) suggests possible simplications in the final result of Gray et al. (2009).

\subsection{Capillary stresses and microstructure}

As a consequence of the result presented in the previous section, Eq. (15) together with (16) lead to the total stress expression for the triphasic REV as:

$$
\begin{aligned}
\boldsymbol{\Sigma}-u_{n} \boldsymbol{\delta}=\boldsymbol{\sigma}^{\mathrm{cont}}-\frac{1}{V} & {\left[u_{c}\left(V_{w} \boldsymbol{\delta}+\int_{S_{s w}} \vec{n} \otimes \vec{x} d S\right)\right.} \\
& \left.+\gamma_{n w}\left(\int_{S_{n w}}(\boldsymbol{\delta}-\vec{n} \otimes \vec{n}) d S+\int_{\Gamma} \vec{\nu}_{n w} \otimes \vec{x} d l\right)\right] \\
\boldsymbol{\sigma}^{\mathrm{cont}}=\frac{1}{V} \sum_{c} \vec{f}^{c} \otimes \vec{l} &
\end{aligned}
$$

The first stress contribution on the r.h.s of Eq. (18) is denoted herein as the contact stress tensor $\sigma^{\text {cont }}$ since it accounts for the stress interaction at any contact $c$ between a solid particle pair 1-2 through the contact force $\vec{f} c$ as sustained by $2, \vec{l}$ being the branch vector from the centre of 1 to the one of 2. This first term describes the part of the solid phase internal forces that is due to the solid phase itself, excluding stress applications by the other phases. In this sense, it has thus been denoted as effective stress by $\mathrm{Lu}$ and Likos (2006); Gray et al. (2009); Li et al. (2016). Also, as a partial confirmation of this denomination, Hicher and Chang (2007); Scholtès et al. (2009); Wan et al. (2015); Duriez and Wan (2016a) considered the above-mentioned stress term to unify the failure description between triphasic and biphasic conditions.

The second stress contribution in Eq. (18) describes the internal forces of a triphasic material due to the capillary pressure $u_{c}$. A spherical stress 
$\left(-u_{c} V_{w} / V \boldsymbol{\delta}\right)$ is due to the fluid volumes and corresponding isotropic pressures. Another contribution encompasses the stress application onto the solid phase by the fluid phases through the suction surfacic loadings along the wetted solid surfaces: $\left(-u_{c} / V \int_{S_{s w}} \vec{n} \otimes \vec{x} d S\right)$. The latter contribution directly depends on the wetted solid surfaces orientations.

Finally, the third stress contribution in Eq. (18) arises from the $\gamma_{n w}$ surface tension forces related to the fluid-fluid interface $S_{n w}$. In particular, the internal forces within the $S_{n w}$ interface are described in terms of $\gamma_{n w}$ and the local interface orientation $(\boldsymbol{\delta}-\vec{n} \otimes \vec{n})$. The stress application by $S_{n w}$ onto the solid particles along the contact lines, depending on the wettability $\vec{\nu}_{n w}$, is also accounted for.

These two former stress contributions coming from the fluid phases and interfaces correspond to the capillary stresses $\sigma^{c a p}$ mentioned in the introduction and defined as:

$$
\sigma^{c a p}=\Sigma-u_{n} \delta-\sigma^{c o n t}
$$

We show herein the capillary stresses depend not only on the fluid pressures $u_{n}, u_{w}$, such as proposed by Bishop (1959): Zienkiewicz et al. (1999): Nuth and Laloui (2008); Boria and Koliii (2009); Lu et al. (2010), but also on the surface tension $\gamma_{n w}$ and more importantly fluid-fluid and solid-fluid interface topologies. Furthermore, we propose a clear microstructure dependency through the following microstructure tensors defined as:

$$
\begin{aligned}
& \boldsymbol{\mu}_{\boldsymbol{V} \boldsymbol{w}}=V_{w} \boldsymbol{\delta} \\
& \boldsymbol{\mu}_{S \boldsymbol{s} \boldsymbol{w}}=\int_{S_{s w}} \vec{n} \otimes \vec{x} d S \\
& \boldsymbol{\mu}_{S n \boldsymbol{w}}=\int_{S n w}(\boldsymbol{\delta}-\vec{n} \otimes \vec{n}) d S \\
& \boldsymbol{\mu}_{\Gamma}=\int_{\Gamma} \vec{\nu}_{n w} \otimes \vec{x} d l
\end{aligned}
$$

such that the capillary stresses express as:

$$
\boldsymbol{\sigma}^{c a p}=-\frac{1}{V}\left[u_{c}\left(\boldsymbol{\mu}_{\boldsymbol{V} \boldsymbol{w}}+\boldsymbol{\mu}_{\boldsymbol{S s \boldsymbol { w }}}\right)+\gamma_{n w}\left(\boldsymbol{\mu}_{\boldsymbol{S n \boldsymbol { w }}}+\boldsymbol{\mu}_{\Gamma}\right)\right]
$$


It is important to recall that a general tensorial (non-spherical) form of the capillary stress is possible due to the nature of all microstructure tensors other than $\boldsymbol{\mu}_{\boldsymbol{V} \boldsymbol{w}}$ in Eq. (25). This general non-spherical form for the capillary stress could not be obtained from a thermodvnamics approach as in Hassanizadeh and Gray (1990); Lu et al. (2010); Nikooee et al. (2013). The shortcoming arises from the constitutive assumptions considered in those works where the free energies are systematically expressed according to scalars such as saturation, whereas tensorial dependencies would be necessary to account for a possibly oriented microstructure.

Being obtained from static considerations without any kinematics, the stress decomposition in Eq. (18) however does not attach any constitutive meaning to the contact and capillary stress tensors. Further investigations are thus led in the remaining developments, where the focus is on the capillary stresses for two extreme saturation regimes.

\subsection{Capillary stresses for high wetting saturation}

In biphasic conditions, the capillary stresses are spherical, with the stress decomposition, Eq. (18), reducing to a form equivalent to the effective stress

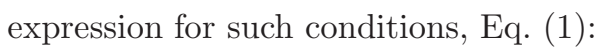

$$
\Sigma=\sigma^{c o n t}+u \delta
$$

with the contact stress tensor $\boldsymbol{\sigma}^{\text {cont }}$ that operates in the solid phase serving as the effective stress for granular materials with negligible solid matrix compressibility.

The same stress decomposition as Eq. (26), together with a spherical nature for the capillary stress, also holds under triphasic conditions in the so-called capillary regime showing high wetting saturations. In this regime, the nonwetting fluid forms isolated bubbles which are of spherical shape to minimize fluid-fluid specific energy. This leads in particular to the following expression for the microstructure tensor $\boldsymbol{\mu}_{\boldsymbol{S n \boldsymbol { w }}}$, obtained from the algebraic equality (17) 
applied to the closed interfaces $S_{n w}$ together with Laplace-Young Eq. (7):

$$
\boldsymbol{\mu}_{\text {Snw }}=\frac{u_{c}}{\gamma_{n w}} V_{n} \boldsymbol{\delta}
$$

such that it is easy to get:

$$
\sigma^{c a p}=-u_{c} \delta
$$

and Eq. (26) with $u=u_{w}$ is recovered, as suggested by Chateau et al. (2002) using a different justification.

The sphericity of capillary stresses in such a saturation regime corresponds to the absence of any preferred orientation at the micro-scale when solid surfaces are completely wetted and $n w$ interfaces are spherical. On the other hand, a preferred orientation is expected for low saturation, in the so-called pendular regime involving oriented capillary bridges.

\section{Multiscale simulation of ideal granular media in the pendular regime}

\subsection{Model formulation}

The DEM model considered herein (Duriez and Wan, 2016a) is built within the code Yade (Šmilauer et al., 2015), being inspired by the one by Scholtès et al. (2009). It applies to low wetting saturations - the so-called pendular regime for which the wetting fluid forms isolated capillary bridges, i.e. menisci between particle pairs (Fig. 3). This saturation regime is of limited extent, up to 5$10 \%$ wetting saturation ratio roughly, but nevertheless provides granular media with very distinct features (e.g. apparent cohesion) compared with biphasic conditions, making this regime worthy of investigation (Chateau et al., 2002; Scholtès et al., 2009; Wan et al., 2015; Wang and Sun, 2015).

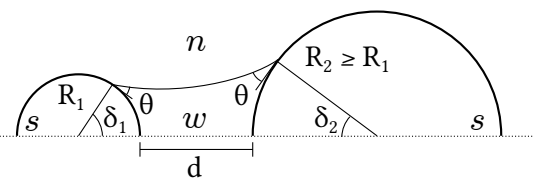

Figure 3: A capillary bridge

The DEM model considers a collection of spherical discrete elements (DE), possibly of different radii $R_{p}$, that correspond in shape and size to the solid 
particles of an idealized granular medium. For such media, the capillary stresses follow directly from Eq. (25) as:

$$
\begin{aligned}
\boldsymbol{\sigma}^{c a p}=-\frac{1}{V} & {\left[u_{c}\left(V_{w} \boldsymbol{\delta}+\sum_{p} R_{p} \int_{S_{p, s w}} \vec{n} \otimes \vec{n} d S\right)\right.} \\
& \left.+\gamma_{n w}\left(\int_{S_{n w}}(\boldsymbol{\delta}-\vec{n} \otimes \vec{n}) d S+\sum_{p} R_{p} \int_{\Gamma_{p}} \vec{\nu}_{n w} \otimes \vec{n} d l\right)\right]
\end{aligned}
$$

Since actual solid particles and DE are quasi-rigid, strains can only arise from particle relative displacements under pair-wise mutual force interactions between the particles.

As a starting point, contacting DE sustain point forces approximating contact interaction following simplified contact laws that express the normal (resp. tangential) contact force as a function of the normal (resp. tangential) relative displacement. In the normal direction, the contact law is linearly elastic in compression and disregards tension. For the tangential direction, the behaviour is governed by Coulombian friction leading to a linear elastic-plastic contact law. Such contact description involves three model parameters, relevant to the contact scale (see Wan et al., 2015, for details).

The next step is to incorporate capillary forces to account for the pore fluid mixture in terms of menisci topology between pairs of particles. A rigorous capillary force determination starts with solving numerically the Laplace-Young Eq. (7) in order to compute the axisymmetric geometry of capillary bridges, gravity being neglected (Fig. 31). The existence and properties of the menisci depend on the particle radii $R_{1}, R_{2}$, their separating distance $d$, the fluid-fluid surface tension, and assumed uniform contact angle and capillary pressure values. The model predicts by design a fluid distribution obeying uniform capillary pressure conditions, as done by Scholtès et al. (2009); Wang and Sun (2015). These uniform suction conditions are specific to thermodynamic equilibrium and the model does not include any pore flow consideration that could address transient phenomena, contrary to Gili and Alonso (2002); Mani et al. (2015); Sivanesapillai et al. (2015). Also, simulations are currently conducted using a constant contact angle irrespective of advancing or receding movements of the 
contact line along the solid surface, thereby neglecting contact angle hysteresis phenomena. However, the model has previously been compared in terms of water retention behavior with experiments (Wan et al., 2015); Duriez and Wan, 2016b).

Once the capillary bridge distribution is determined, attractive capillary forces $\vec{f}^{c a p}$ between capillary bonded particle pairs are finally obtained from the capillary pressure and fluid-fluid surface tension loadings on either spherical particle:

$$
\begin{aligned}
& \left\|\vec{f}^{c a p}\right\|=\pi R_{1} \sin \delta_{1}\left(u_{c} R_{1} \sin \delta_{1}+2 \gamma_{n w} \sin \left(\theta+\delta_{1}\right)\right) \\
& =\pi R_{2} \sin \delta_{2}\left(u_{c} R_{2} \sin \delta_{2}+2 \gamma_{n w} \sin \left(\theta+\delta_{2}\right)\right)
\end{aligned}
$$

with $\delta_{1}, \delta_{2}$ the filling angles on each particle 1,2 (Fig. 3). Considering either particle to compute the capillary force norm - using index 1 or 2 in Eq. (30) - is immaterial due to the meniscus equilibrium.

In line with the previous focus on solid-fluid surface tensions, it is worth to clarify that these do not contribute to the capillary force. A first possible justification to this assertion is to consider the solid-fluid surface tensions as an internal force to the solid particles, thus leading to no resultant external forces on the DE. Alternatively, in case the DE were assimilated to solid particles separately from the solid-fluid interfaces, two external loadings proportional to $\gamma_{s \alpha}, \alpha=n ; w$ would apply on the DE:

1. a first one along $S_{s \alpha}: \int_{S_{s \alpha}}-\gamma_{s \alpha} \operatorname{div}_{\mathrm{S}}(\vec{n}) \vec{n} d S$, see Eq. (8)

2. a second one along the contour $C$ of $S_{s \alpha}: \int_{C} \gamma_{s \alpha} \vec{\nu}_{s \alpha} d l$, see Eq. (9) and the resulting loading would be zero, see Eq. (5) and also Lunati (2007); Shikhmurzaev (2008). The absence of solid-fluid surface tension $\gamma_{s \alpha}$ from the resultant forces used in DEM to describe internal forces is consistent- though not equivalent - with their zero contribution to the total stresses of a triphasic material, discussed in Section 2.2 .

Through the capillary force determination, the particle size distribution, the fluid-fluid surface tension and the contact angle constitute additional model parameters (Duriez and Wan, 2016a). 


\subsection{Numerical description of stress}

According to their calculation paradigm, DEM models of multiphasic conditions (Gili and Alonso, 2002; Scholtès et al., 2009; Richefeu et al., 2006) including the present one describe all internal forces of a triphasic granular medium exclusively with interaction forces between DE. In particular, non-solid phases with their internal forces are not directly included, but rather reduced to the resultant forces induced on the solid phase. A total stress tensor is then defined for the DEM sample from contact and capillary forces for all contacts $c$ and menisci $m$, applying the classical homogenization techniques to the DE (Duriez and Wan, 2016c):

$$
\begin{aligned}
& \boldsymbol{\Sigma}=\frac{1}{V} \sum_{D E} \int_{V_{D E}} \boldsymbol{\sigma} d V=\frac{1}{V} \sum_{c} \vec{f}^{c} \otimes \vec{l}+\frac{1}{V} \sum_{m} \vec{f}^{c a p} \otimes \vec{l}=\boldsymbol{\sigma}^{c o n t}+\boldsymbol{\sigma}_{D E M}^{c a p} \\
& \boldsymbol{\sigma}_{\boldsymbol{D E M}}^{\mathrm{cap}}=\frac{1}{V} \sum_{m} \vec{f}^{c a p} \otimes \vec{l}
\end{aligned}
$$

By contrast with the computation of particles relative displacements and strains in granular media, the sole consideration of resultant forces may be inadequate to describe stresses in a general manner, as evidenced by any solid sustaining non-zero stresses while in equilibrium. For instance, the DEM is unable to capture the stress state of a biphasic granular media showing a uniform fluid pressure because the fluid-induced resultant force on solid particles is zero in such case. For this reason, the DEM total stress in Eq. (31) is to be compared with the net stress $\left(\boldsymbol{\Sigma}-u_{n} \boldsymbol{\delta}\right)$ in Eq. (18) derived from analytical homogenization.

The current stress analysis of the DEM model involves the same contact stress tensor $\sigma^{\text {cont }}$ as the one derived previously from analytical homogenization, Eq. (19). As for the fluid mixture stress contribution, the DEM framework leads to a capillary stress expression from resultant capillary forces, clearly distinct in nature from the previous expression obtained from analytical homogenization, Eq. (29). As such, we refer to the capillary stress tensor defined by Eq. (32) as $\boldsymbol{\sigma}_{\boldsymbol{D E M}}^{c a p}$, as opposed to the previous capillary stress tensor $\sigma^{\text {cap }}$ expressed by Eq. (29). Nevertheless, Chateau et al. (2002) suggested with little justification that a stress description from resultant forces is still possible, such 
that $\sigma^{c a p}=\sigma_{D E M}^{c a p}$. Duriez and Wan (2016b d) presented numerical comparisons confirming it, provided the fluid-fluid interface is accounted for in addition to bulk phases (Wan et al., 2015). In order to further assess the equivalence of the stress state of DEM models with the stress state of simulated triphasic materials, as derived from homogenization, the equality of the first invariants of $\sigma^{c a p}$ and $\sigma_{D E M}^{c a p}$ is herein demonstrated from the Laplace-Young equation as follows.

The mean capillary stress as predicted by the homogenization approach, denoted as $p_{\text {hom }}^{c a p}$, is obtained taking the trace of Eq. (29):

$$
p_{\text {hom }}^{\text {cap }}=-\frac{1}{3 V}\left[u_{c}\left(3 V_{w}+\sum_{p} R_{p} S_{p, s w}\right)+\gamma_{n w}\left(2 S_{n w}+\sum_{p} R_{p} \Gamma_{p} \sin \theta\right)\right]
$$

By construction of the DEM model, the comparison is restricted to the pendular regime for which the wetting fluid forms menisci between particle pairs $(i, j)=$ $(1,2)$. Then, Eq. (33) is rewritten accounting for menisci microstructure:

$p_{\text {hom }}^{\text {cap }}=-\frac{1}{3 V} \sum_{m}\left[u_{c}\left(3 V_{m}+\sum_{i=1,2} R_{i} S_{i, s w}\right)+\gamma_{n w}\left(2 S_{m}+\sum_{i=1,2} R_{i} \Gamma_{i} \sin \theta\right)\right]$

For a given meniscus of volume $V_{m}$ and fluid-fluid surface $S_{m}$, the wetted surface $S_{i, s w}$ on each spherical particle $i$ is $S_{i, s w}=2 \pi R_{i}{ }^{2}\left(1-\cos \delta_{i}\right)$ and the meniscus contour $\Gamma_{i}$ is equal to $2 \pi R_{i} \sin \delta_{i}$, see Fig. 3.

On the other hand, the mean stress $p_{D E M}^{c a p}$ of $\boldsymbol{\sigma}_{\boldsymbol{D E M}}^{c a p}$ involves the scalar products $\vec{f}^{c a p} . \vec{l}$ through the trace of $\vec{f}^{c a p} \otimes \vec{l}$, see Eq. (32), such that:

$$
p_{D E M}^{c a p}=-\frac{1}{3 V} \sum_{m}\left(u_{c} \pi R_{1}^{2} \sin ^{2} \delta_{1}+\gamma_{n w} \Gamma_{1} \sin \left(\theta+\delta_{1}\right)\right)\left(R_{1}+R_{2}+d\right)
$$

Comparing the two expressions of the mean capillary stress, qualitative differences clearly appear with the menisci surface $S_{m}$ and volume $V_{m}$ entering Eq. (34) and not (35) for instance. However, using the Laplace-Young equation and applying Eq. (16) to the bridge surface $S_{m}$, Appendix B demonstrates that 
for any meniscus:

$$
\begin{aligned}
& u_{c}\left(3 V_{m}+\sum_{i=1,2} R_{i} S_{i, s w}\right)+\gamma_{n w}\left(2 S_{m}+\sum_{i=1,2} R_{i} \Gamma_{i} \sin \theta\right) \\
& =\left(u_{c} \pi R_{1}^{2} \sin ^{2} \delta_{1}+\gamma_{n w} \Gamma_{1} \sin \left(\theta+\delta_{1}\right)\right)\left(R_{1}+R_{2}+d\right)
\end{aligned}
$$

Eq. (36) is a general property of capillary bridges between spherical particles, under mechanical equilibrium conditions i.e. complying with the Laplace-Young equation. It could be used as such to validate triphasic microstructure experimental measurements at the capillary bridge scale. Used here at the REV scale, it remarkably proves that the mean capillary stresses provided by DEM modelling or analytical homogenization are finally equivalent, validating the stress description by DEM in spite of the above mentionned possible reasons for discrepancy.

From a numerical point of view, the complete equality between $\sigma_{D \boldsymbol{D} \boldsymbol{M}}^{c a p}$ and $\boldsymbol{\sigma}^{c a p}$, considering other invariants than $p^{c a p}$ as well as principal directions, has been recently obtained by Duriez and Wan (2016b).

\section{Microstructure and anisotropy of capillary stresses in the pendular regime}

The DEM model is finally applied to a slightly polysized numerical packing of spherical particles. Table 1 presents the retained parameters.

Table 1: DEM model parameters

\begin{tabular}{|c|c|c|c|c|c|c|c|}
\hline \multicolumn{3}{|c|}{ Contact } & \multicolumn{3}{c|}{ Packing } & \multicolumn{2}{c|}{ Capillarity } \\
\hline$k_{n} / D$ & $k_{t} / k_{n}$ & $\varphi$ & $D_{\min }$ & $D_{\max }$ & $e$ & $\gamma_{n w}$ & $\theta$ \\
$(\mathrm{MPa})$ & $(-)$ & $\left(^{\circ}\right)$ & $(\mathrm{mm})$ & $(\mathrm{mm})$ & $(-)$ & $(\mathrm{N} / \mathrm{m})$ & $\left(^{\circ}\right)$ \\
10 & 0.21 & 20 & 0.25 & 0.3 & 0.61 & 0.073 & 10 \\
\hline
\end{tabular}

Three mechanical parameters, $k_{n} / \bar{D}, k_{t} / k_{n}$, and $\varphi$, are relevant to the two constant local stiffnesses $k_{n}, k_{t}$ and the local friction angle $\varphi$ of the contact interaction, as detailed by Wan et al. (2015). Their values are taken from comparable DEM simulations on glass beads (Hanes and Walton, 2000; Favier et al., 2009). Regarding the capillary interaction, the particle size distribution is chosen to be uniform in number between two extreme diameters $D_{\min }, D_{\max }$. 
The void ratio $e$ of the considered packing is such that the sample shows a dense, i.e. dilatant behaviour under shearing for the considered confining pressures. Finally, an air-water fluid mixture is considered with the relevant surface tension value at ambient temperature and a small, though not zero, contact angle value $\theta$, in accordance with the wetting of glass beads by water (Richefeu et al.,, 2006; Scheel et al., 2008).

\subsection{Isotropic solid packing}

An isotropic packing is first considered with the numerical sample being under hydrostatic total stresses (with $\Sigma_{x x}=\Sigma_{y y}=\Sigma_{z z}=10 \mathrm{kPa}$ ) and various capillary pressure, i.e. wetting saturation $S_{r}$ values. According to the model design presented back in Section 3.1, wetting saturation (capillary pressure) is uniformly imposed to the sample: capillary bridges are introduced solving the Laplace-Young equation between particles pairs for a given capillary pressure value. Doing so, capillary bridges are introduced between both contacting and separated particles, up to some interparticle distance beyond which the LaplaceYoung equation does not give any solution. Due to the isotropic configuration of the solid phase, the resulting fluid phases and interfaces distributions are also isotropic and any microstructure tensor $\boldsymbol{\mu}$ among $\boldsymbol{\mu}_{\boldsymbol{V} \boldsymbol{w}}, \boldsymbol{\mu}_{\boldsymbol{S s \boldsymbol { w }}}, \boldsymbol{\mu}_{\boldsymbol{S n \boldsymbol { w }}}, \boldsymbol{\mu}_{\Gamma}$ is spherical, i.e.

$$
\boldsymbol{\mu}=m \boldsymbol{\delta}
$$

with $m$ defined as the first invariant of $\boldsymbol{\mu}$ :

$$
m=\frac{\operatorname{tr}(\boldsymbol{\mu})}{3}
$$

and being expressed for the various $\boldsymbol{\mu}$ tensors as:

$$
\begin{aligned}
m_{V w} & =V_{w} \\
m_{S s w} & =\frac{1}{3} \sum_{p} R_{p} S_{p, s w} \\
m_{S n w} & =\frac{2}{3} S_{n w} \\
m_{\Gamma} & =\frac{1}{3} \sum_{p} R_{p} \Gamma_{p} \sin \theta
\end{aligned}
$$


Thus, the capillary stresses $\boldsymbol{\sigma}^{\text {cap }}$ reduce to their spherical part $p^{c a p} \boldsymbol{\delta}$, with $p^{c a p}$ previously expressed according to the microstructure in Eq. (33):

$$
\boldsymbol{\sigma}^{c a p}=-\frac{1}{V}\left[u_{c}\left(m_{V w}+m_{S s w}\right)+\gamma_{n w}\left(m_{S n w}+m_{\Gamma}\right)\right] \boldsymbol{\delta}=p^{c a p} \boldsymbol{\delta}
$$

The capillary stress together with its distinct contributions are depicted according to the wetting saturation in Fig. 5 , based on microstructural information

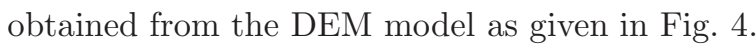

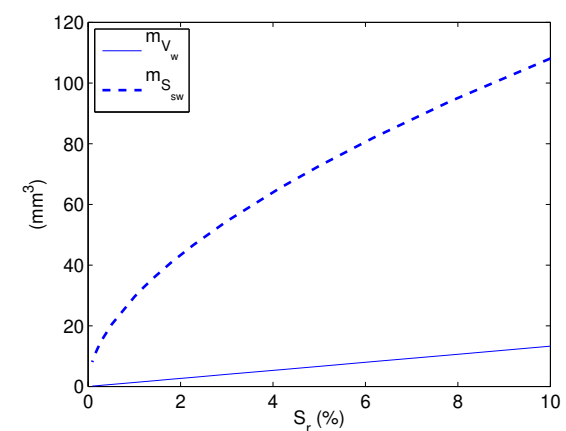

(a) $\boldsymbol{\mu}_{\boldsymbol{V} \boldsymbol{w}}=m_{V w} \boldsymbol{\delta}$ and $\boldsymbol{\mu}_{S \boldsymbol{s} \boldsymbol{w}}=m_{S s w} \boldsymbol{\delta}$ relevant to wetting volume and wetted solid surfaces

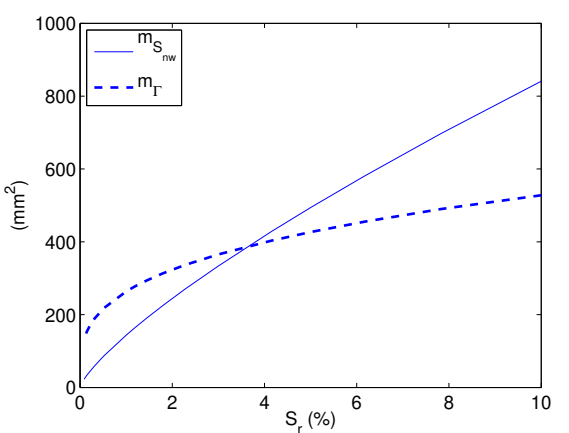

(b) $\boldsymbol{\mu}_{\text {Snw } \boldsymbol{w}}=m_{S n w} \boldsymbol{\delta}$ and $\boldsymbol{\mu}_{\Gamma}=m_{\Gamma} \boldsymbol{\delta}$ relevant to $n w$ interfaces and contact lines

Figure 4: Scalar representation of spherical microstructure tensors during the hydraulic loading of an isotropic sample

The capillary stress shows a non-zero limit when the saturation asymptotically tends to zero, while it is strictly zero for the dry case. This non-zero limit is related to the non-zero limit of the capillary force for a capillary bridge (between contacting particles) whose volume asvmptotically vanishes (Gili and Alonso, 2002; Herminghaus, 2005). For such infinitesimal saturations, the capillary stress mostly depends on the capillary pressure and the minutely wetted solid surfaces, the other stress contributions being negligible. For higher saturations close to the upper limit of the pendular regime, a significant contribution from surface tension arises through fluid-fluid interfaces and contact lines, see Fig. 5(a) Generally speaking, and as it is obvious for the contact line term, the relative weight of the distinct contributions depends on the wetting angle 


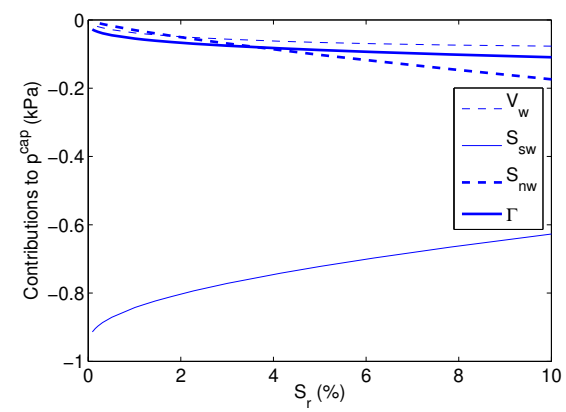

(a) Contributions from the wetting fluid volume $V_{w}$, the wetted surfaces $S_{s w}$, the interface $S_{n w}$ and the contact lines $\Gamma$, see Eq. (43)

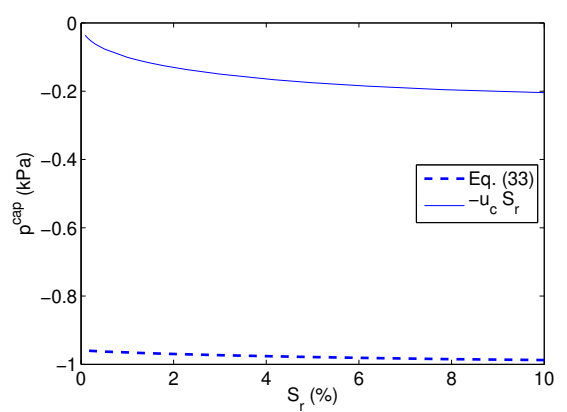

(b) Mean capillary stress from microstructure, Eq. 33), compared with $-u_{c} S_{r}$

Figure 5: Capillary stresses during the hydraulic loading of an isotropic sample

$\theta$ (Duriez and Wan, 2016a). In total the capillary stress as obtained from micromechanics is little affected by the considered changes in saturation, Fig. 5(b) Even though an increasing saturation magnifies the relevant micro-features, e.g. the wetted surfaces (Fig. (4), it is accompanied by a decrease in capillary pressure which directly enters the capillary stress expression, Eq. (43).

Also, the mean capillary stress as obtained here from micromechanics is compared with the classical expression $p^{c a p}=-u_{c} S_{r}$. The latter expression corresponds to the early proposal by Bishop (1963), taking the corresponding $\chi$ parameter equal to saturation $S_{r}$. This expression has more recently also been proposed from thermodynamical considerations (Hassanizadeh and Grav, 1990; Lu et al., 2010). However, such an expression significantly underestimates the capillary stresses in the pendular regime, as compared with the present micromechanical approach, see Fig. 5(b), The discrepancy is believed to arise because of the missing microstructure dependencies in the constitutive assumptions formulated in such thermodynamic approaches. It should be noted that Nikooee et al. (2013) later on introduced new dependencies in the constitutive assumptions made by Hassanizadeh and Gray (1990), modifying the initial result. 


\subsection{Anisotropic solid packing}

A second configuration is herein considered such as the classical critical state which is reached after sustained shearing of a granular material. Here, the numerical sample is brought to critical state by imposing an axisymmetric, socalled "triaxial", compression up to $45 \%$ axial strain $\varepsilon_{1}=\varepsilon_{y y}$, under $10 \mathrm{kPa}$ constant lateral pressure and dry conditions.

At this stage, an anisotropic contact network has been induced by the deviatoric loading, starting from an initially isotropic configuration. Imposing subsequently triphasic conditions to the sample with various capillary pressure values, and solving for capillary bridges between all contacting and separated particles, an anisotropic liquid distribution arises since capillary bridges preferably form between close particles. The microstructure tensors then may show both a spherical and deviatoric component, $\boldsymbol{\mu}_{\boldsymbol{V} \boldsymbol{w}}$ excepted. Taking advantage of axisymmetry and by analogy to the classical stress variables $p=\left(\Sigma_{x x}+\Sigma_{y y}+\Sigma_{z z}\right) / 3$ and $q=\Sigma_{y y}-\Sigma_{x x}=\Sigma_{y y}-\Sigma_{z z}$, the spherical and deviatoric part of any microstructure tensor $\boldsymbol{\mu}$ are respectively quantified by the scalars $m$, Eq. (38), and $t$ defined as:

$$
t=\sqrt{\frac{3}{2}}\|\boldsymbol{\mu}-m \boldsymbol{\delta}\|=\mu_{y y}-\mu_{x x}=\mu_{y y}-\mu_{z z}
$$

where, obviously:

$$
t_{V w}=0
$$

For this critical state configuration, deviatoric components are measured as expected for some microstructure tensors (Fig. 6), namely $\boldsymbol{\mu}_{S \boldsymbol{s} \boldsymbol{w}}$ and $\boldsymbol{\mu}_{\Gamma}$ that refer to the wetted solid surfaces and contact lines, respectively.

The deviatoric nature of $\boldsymbol{\mu}_{\boldsymbol{S s w}}$, as measured by $t_{S s w} / m_{S s w}$, can be compared to the fabric tensor $\boldsymbol{F}$ that describes the contact network:

$$
\boldsymbol{F}=\frac{1}{n_{c}} \sum_{\text {cont. }} \vec{n} \otimes \vec{n}
$$

where $n_{c}$ is the total number of contacts between spherical particles, each of them being oriented by $\vec{n}$. An exact equality between $t_{F} / m_{F}=3 t_{F}$ and $t_{S s w} / m_{S s w}$ would hold for a monosized solid packing under very high capillary 


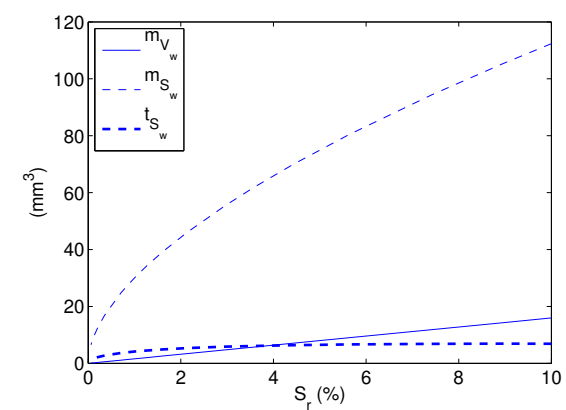

(a) $\boldsymbol{\mu}_{\boldsymbol{V} \boldsymbol{w}}$ and $\boldsymbol{\mu}_{S \boldsymbol{s} w}$ relevant to wetting volume and wetted solid surfaces

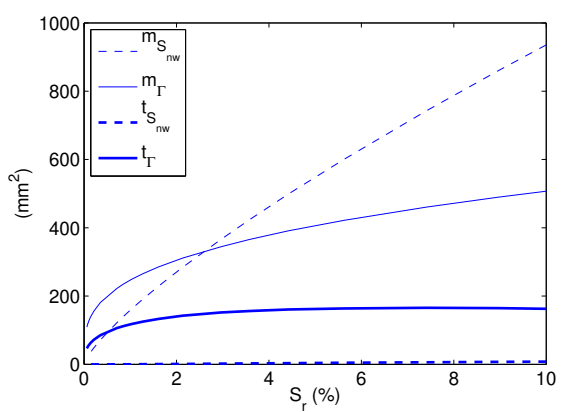

(b) $\mu_{S n w}$ and $\mu_{\Gamma}$ relevant to $n w$ interfaces and contact lines

Figure 6: Scalar representations of axisymmetric microstructure tensors during the hydraulic loading of a sample at critical state: spherical $(m)$ and deviatoric $(t)$ parts

pressure for which capillary bridges exist at contacts only and show negligible wetted surfaces. Here, the discrepancy between the two ratios narrows to $11 \%$ for $u_{c}=40 \mathrm{kPa}$ and $S_{r} \approx 0.063 \%$ (Fig. [7). For higher saturations when cap-

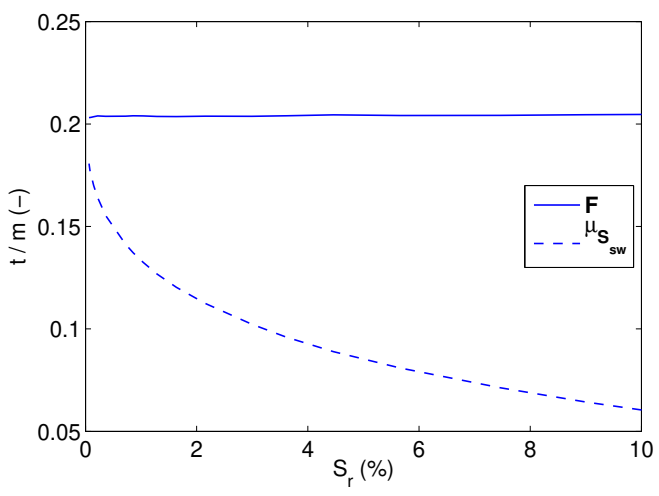

Figure 7: Deviatoric natures of the fabric tensor $\boldsymbol{F}$ and the wetted solid surfaces microstructure tensor $\boldsymbol{\mu}_{\boldsymbol{S} \boldsymbol{s} \boldsymbol{w}}$

illary bridges between separated particles exist, the deviatoric nature of $\boldsymbol{\mu}_{\boldsymbol{S} \boldsymbol{s} w}$ decreases while the anisotropy of the solid packing i.e. $t_{F}$ is barely affected by the hydraulic loading. In fact, deviatoric strains and changes in $t_{F}$ arise transitioning from dry to unsaturated conditions but become negligible upon 
subsequent hydraulic loading in triphasic conditions (see also Wan et al., 2015).

Focusing now on the the fluid-fluid interface, the microstructure tensor $\boldsymbol{\mu}_{S n \boldsymbol{w}}$ interestingly turns out to be spherical with a very good approximation. At the capillary bridge scale, a deviatoric nature exists for $\boldsymbol{\mu}_{\text {Snw }}$ (Fig. 8 and 9(a), which is in relationship with an oriented meniscus shape. However, these devi-

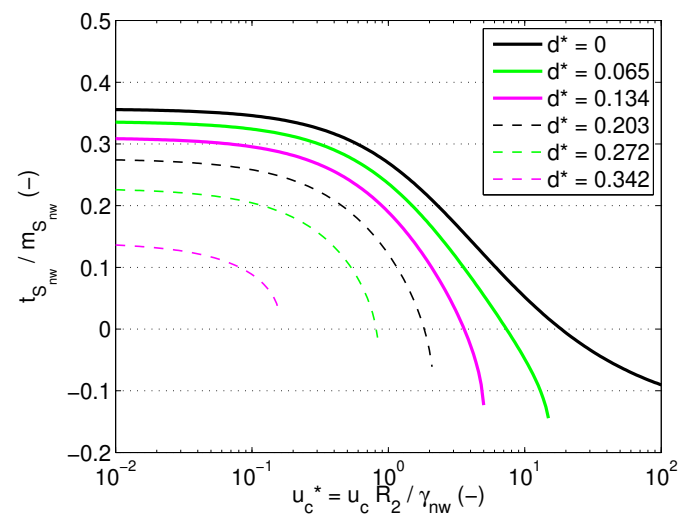

Figure 8: Deviatoric nature of $\boldsymbol{\mu}_{\boldsymbol{S} \boldsymbol{n} \boldsymbol{w}}$ for capillary bridges between two particles showing $R_{2} / R_{1}=1.1$, considering various dimensionless capillary pressures $u_{c}^{*}$ and interparticle distance $d^{*}=d / R_{2}$

atoric components approximatively cancel each other at the REV scale (Fig.6(b) and $9(\mathrm{~b})$. The sphericity of $\boldsymbol{\mu}_{\boldsymbol{S n} \boldsymbol{w}}$ at the macro-scale has also been observed by Duriez and Wan (2016b) for other numerical samples, still in the pendular regime. This sphericity of the $n w$ interface was previously proposed as an assumption by Grav et al. (2009), without any possible measures to support it.

Finally, the capillary stresses depending on $\boldsymbol{\mu}_{\boldsymbol{V} \boldsymbol{w}}, \boldsymbol{\mu}_{\boldsymbol{S s \boldsymbol { w }}}, \boldsymbol{\mu}_{\boldsymbol{S n \boldsymbol { w }}}, \boldsymbol{\mu}_{\Gamma}$ deviate from an averaged fluid pressure (Fig. 10) showing a non-zero deviatoric component $q^{\text {cap }}$ defined as:

$$
q^{c a p}=\sigma_{y y}^{c a p}-\sigma_{x x}^{c a p}=\sigma_{y y}^{c a p}-\sigma_{z z}^{c a p}=-\sqrt{\frac{3}{2}}\left\|\boldsymbol{\sigma}^{c a p}-p^{c a p} \boldsymbol{\delta}\right\|
$$

For very low saturations, deviatoric capillary stresses arise mostly from the capillary pressure internal forces along the wetted solid surfaces, in relationship with the anisotropic solid packing as discussed in the above. For higher saturations, surface tension internal forces along the menisci contours contribute 


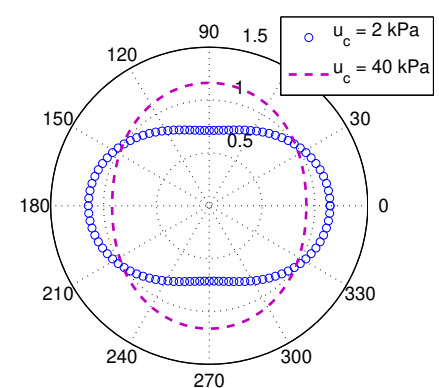

(a) At the capillary bridge scale for two contacting particles with $D_{\max }$ and $D_{\min }$ diameters, $\alpha$ being measured from the contact direction

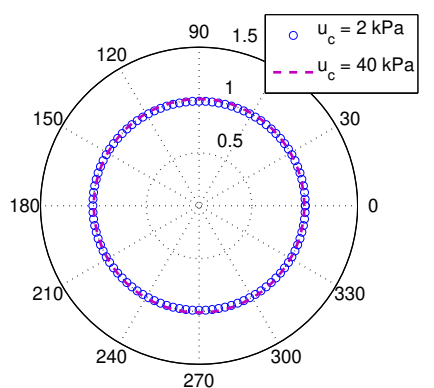

(b) At the sample scale at critical state, $\alpha$ being measured from the major principal total stress direction

Figure 9: Orientation of the $n w$ interface at different scales and for different capillary pressures: polar representation of $3 / S_{n w} \vec{v}(\alpha) \cdot\left(\int_{S_{n w}} \vec{n} \otimes \vec{n} d S\right) \vec{v}(\alpha), \vec{v}$ being an arbitrary unit vector oriented by the polar angle $\alpha$

also significantly to deviatoric capillary stresses, see Fig. 10(a) Obviously, the classical expression $\boldsymbol{\sigma}^{\text {cap }}=-u_{c} S_{r} \boldsymbol{\delta}$ is inadequate to describe such deviatoric capillary stresses, in addition to underestimate the mean capillary stress $p^{c a p}$ in this anisotropic configuration, see Fig. 10(b), in line with the previous comparison for an isotropic case, Fig. $5(\mathrm{~b})$.

\subsection{Evolving solid packing}

Finally, the case of a solid packing subject to important deformations is tackled considering the following mechanical loadings in triphasic conditions: two triaxial compressions under 4 and $40 \mathrm{kPa}$ of capillary pressure and $10 \mathrm{kPa}$ confining pressure. Such mechanical loading is considered to occur within smaller time scales than the hydraulic loadings previously considered under thermodynamic equilibrium. As such, the possibility to solve the Laplace-Young equation is complemented by additional conditions before considering capillary bridges. Namely, capillary bridges are now assumed to form between contacting particles only, in accordance with observations by Herminghaus (2005). In case particles subsequently separate because of the mechanical loading, the menisci continue 


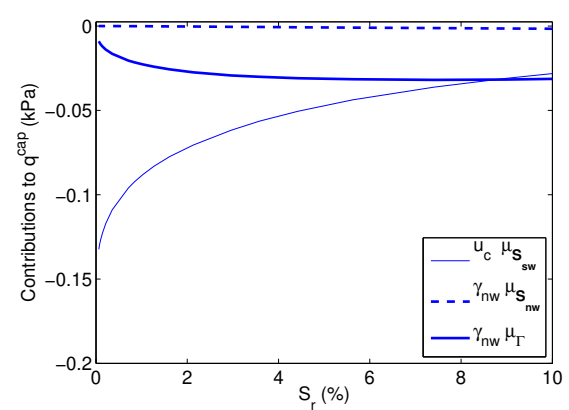

(a) Contributions from $S_{s w}, S_{n w}$ and $\Gamma$ to $q^{\text {cap }}$

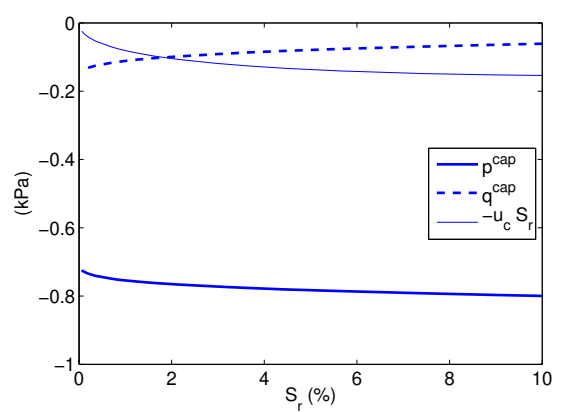

(b) Capillary stresses, compared with $u_{c} S_{r}$

Figure 10: Capillary stresses during hydraulic loading of a sample at critical state

to be considered as long as the interparticle distance allows for a solution of the Laplace-Young equation. These processes of bridge creation and rupture occur in a fully drained manner, and during the mechanical loading, the saturation slightly evolves in $[2.8 \% ; 3.6 \%]$ and $[0.06 \% ; 0.09 \%]$ for a 4 and $40 \mathrm{kPa}$ capillary pressure, respectively. Similar macroscopic behaviours are obtained during the two loading paths, see Fig. 11(a) the capillary stresses being a small portion of the total stresses (Fig. 11). This is due to the particle size distribution that confers the numerical sample a limited sensitivity to triphasic conditions: the latter would be greater for smaller particles.

Starting from an isotropic initial state, the solid phase turns anisotropic because of the deviatoric mechanical loading, as evidenced by the fabric tensor $\boldsymbol{F}$, see Fig. 12(a), As observed during hydraulic loadings in the above, the wetted solid surfaces tend to follow the same anisotropy as the solid phase, especially for higher capillary pressure, see Fig. 12(a) As for the fluid-fluid interface, the sphericity of the corresponding microstructure tensor $\boldsymbol{\mu}_{\boldsymbol{S n \boldsymbol { w }} \boldsymbol{w}}$ is also verified along this mechanical loading, see Fig. 12(b), Note finally that the contact line microstructure tensor $\boldsymbol{\mu}_{\boldsymbol{\Gamma}}$ is the one showing the greatest deviatoric nature as measured by $t / m$ because of the low contact angle that is here considered.

Because of processes involving microstructure orientation, deviatoric capil- 


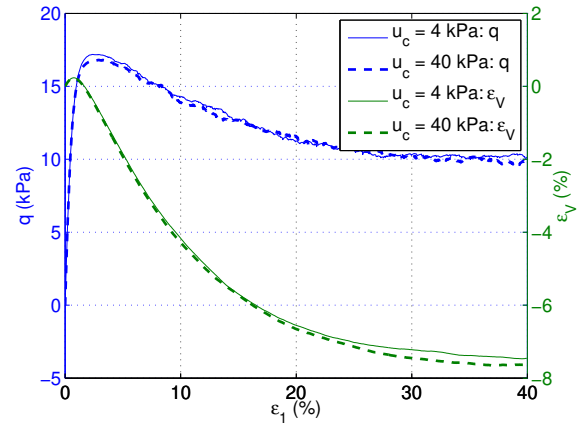

(a) Macroscopic behavior $q\left(\varepsilon_{1}\right)$ and $\varepsilon_{V}\left(\varepsilon_{1}\right)$, with $\varepsilon_{V}=\varepsilon_{y y}+2 \varepsilon_{x x}=\varepsilon_{y y}+2 \varepsilon_{z z}$

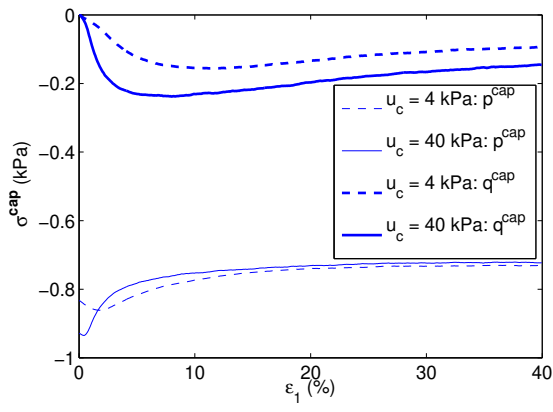

(b) Capillary stresses

Figure 11: Triaxial compression loading paths in triphasic conditions

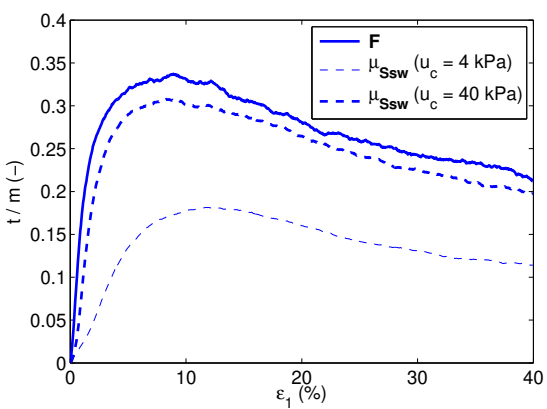

(a) Deviatoric nature of $\boldsymbol{F}$ and $\boldsymbol{\mu}_{S \boldsymbol{s} \boldsymbol{w}}$

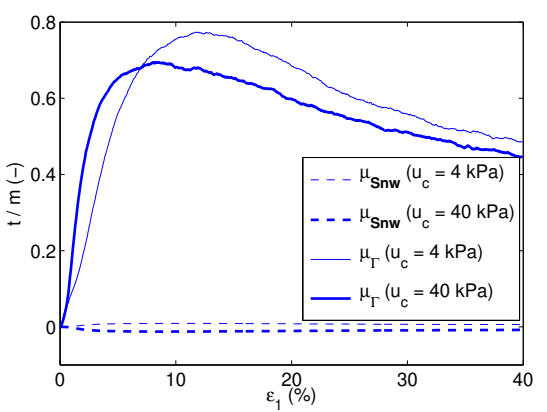

(b) Deviatoric nature of $\boldsymbol{\mu}_{\boldsymbol{S n} \boldsymbol{w}}$ and $\boldsymbol{\mu}_{\Gamma}$

Figure 12: Microstructure evolutions during the triaxial compressions 
lary stresses arise during the mechanical loading, see Fig. 11(b). The largest (in absolute value) deviatoric capillary stresses are obtained before the critical state previously analyzed. They occur at the beginning of the total stress softening when the microstructure anisotropy is the most developed (Fig. 12).

Let us finally recall that a failure criterion encompassing stress limit states for granular materials in both dry and wet conditions can be expressed according to the contact stress tensor $\boldsymbol{\sigma}^{\text {cont }}=\boldsymbol{\Sigma}-u_{n} \boldsymbol{\delta}-\boldsymbol{\sigma}^{\text {cap }}$ (Hicher and Chang, 2007; Scholtès et al., 2009; Wan et al., 2015; Duriez and Wan, 2016a). For axisymmetric loading paths such as triaxial compressions, it is sufficient to consider the stress ratio $q^{\text {cont }} / p^{\text {cont }}$ that obeys:

$$
\frac{q^{\text {cont }}}{p^{\text {cont }}}=\frac{q-q^{\text {cap }}}{p-u_{n}-p^{c a p}}
$$

Obviously, predicting failure for a given external loading in terms of the total stresses $p, q$ requires a precise estimation of both $p^{c a p}$ and $q^{c a p}$. Neglecting in particular the deviatoric capillary stress $q^{\text {cap }}$ could lead to underestimating failure.

\section{Conclusion}

The present micromechanical analysis elucidates the nature of stresses in triphasic granular materials by accurately taking into account details of internal forces within the three bulk phases and the three associated interfaces. It is in particular demonstrated analytically that solid-fluid surface tensions do not contribute to the average stress whenever solid contact surfaces are negligible.

The proposed tensorial stress decomposition involves a capillary stress that is derived in terms of capillary pressure, fluid-fluid surface tension, as well as other tensorial quantities that depend on microstructure. An important implication of this expression is that the mixture of the two non-viscous fluids is able to sustain deviatoric stresses in triphasic media as demonstrated in DEM numerical experiments that precisely describe the micro-scale. Numerical experiments considered idealized spherical granular materials. If the particle shape of real granular materials were to be considered, it would be expected that the 
anisotropy of the capillary stress due to the fluid mixture would be even greater than in the present analysis.

Another major finding of the present work is that the DEM stress description from resultant forces acting on solid particles is equivalent in the pendular regime to the stress decomposition herein proposed from analytical homogenization of all phases and interfaces. The demonstration of the DEM stress description for granular media under low-saturation fully validates its use under such wet conditions. This also implies that possible simplifications in the realm of microstructure experimental measurements can be made by using Eq. (36) that expresses complex relationships between micro-scale parameters.

Contrary to alternate approaches based on thermodynamics, the proposed stress decomposition does not attach any constitutive meaning to the distinct stress contributions since only statical considerations are made without any kinematics. On the other hand, and with respect to Hassanizadeh and Grav (1990); Grav et al. (2009); Madeo et al. (2013); Nikooee et al. (2013) who considered an elastic behaviour for the solid phase, the present result is not restricted to any particular mechanical behaviour of the different phases - except that fluid viscosity was neglected. Comparing the present work with thermodynamics approaches allows us to assess how well stress decompositions obtained from thermodynamics account for the microstructure of triphasic granular materials, which is believed to be of utmost importance. In fact, it is believed that tensorial free energy dependencies, such as the microstructure tensors proposed here, should be introduced in addition to scalar dependencies to capture the deviatoric nature of capillary stresses from thermodynamics.

Ongoing work is actually being pursued in order to assess the effective nature of $\boldsymbol{\sigma}^{\text {cont }}=\boldsymbol{\Sigma}-u_{n} \boldsymbol{\delta}-\boldsymbol{\sigma}^{\text {cap }}$ from a constitutive point of view, and not restricted to failure description. In the meantime, the proposed stress decomposition already sheds further light on the old debate regarding the validity of Bishop's effective stress equation in partially saturated soils in that it identifies missing microstructural dependencies in Bishop's capillary stress equation. 


\section{Appendix A: Demonstration of Eq. (16)}

Stokes' theorem states the surface integral of the curl of an arbitrary vector field $\vec{F}$ over a surface $S$ equals to the line integral of that vector field over the surface's boundary $\Gamma$. Adopting $\overrightarrow{d l}=\vec{h} d l$ and the index notation with Einstein convention:

$$
\begin{gathered}
\int_{S} \vec{\nabla} \times \vec{F} \cdot \vec{n} d S=\int_{\Gamma} \vec{F} \cdot \overrightarrow{d l} \\
\Leftrightarrow \int_{S} \epsilon_{i j k} \frac{\partial F_{k}}{\partial x_{j}} n_{i} d S=\int_{\Gamma} F_{i} h_{i} d l
\end{gathered}
$$

In Eq. (A.1), $\epsilon_{i j k}$ is the Levi-Civita symbol. It is possible to replace the vector field $\vec{F}$ with a second-order tensor field $\boldsymbol{W}$ to get:

$$
\int_{S} \epsilon_{i j k} \frac{\partial W_{m k}}{\partial x_{j}} n_{i} d S=\int_{\Gamma} W_{m i} h_{i} d l
$$

It is convenient here to define $\boldsymbol{W}$ from the Levi-Civita symbol, a second order tensor field $\boldsymbol{w}$ and a constant vector $\vec{b}: W_{i j}=\epsilon_{j n l} w_{i n} b_{l}$. Then, the 1.h.s of Eq. (A.2) is:

$$
\begin{aligned}
\int_{S} \epsilon_{i j k} \frac{\partial W_{m k}}{\partial x_{j}} n_{i} d S & =\int_{S} \epsilon_{i j k} \epsilon_{k n l}\left(\frac{\partial w_{m n}}{\partial x_{j}} b_{l}+\frac{\partial b_{l}}{\partial x_{j}} w_{m n}\right) n_{i} d S \\
& =\int_{S} \epsilon_{i j k} \epsilon_{k n l} \frac{\partial w_{m n}}{\partial x_{j}} b_{l} n_{i} d S
\end{aligned}
$$

Using the identity:

$$
\epsilon_{i j k} \epsilon_{k n l}=\epsilon_{k i j} \epsilon_{k n l}=\delta_{i n} \delta_{j l}-\delta_{i l} \delta_{j n}
$$

we get finally for the l.h.s of (A.2):

$$
\begin{aligned}
\int_{S} \epsilon_{i j k} \frac{\partial W_{m k}}{\partial x_{j}} n_{i} d S & =\int_{S}\left(\frac{\partial w_{m i}}{\partial x_{j}} b_{j}-\frac{\partial w_{m j}}{\partial x_{j}} b_{i}\right) n_{i} d S \\
& =b_{k} \int_{S}\left(\frac{\partial w_{m i}}{\partial x_{k}}-\frac{\partial w_{m j}}{\partial x_{j}} \delta_{i k}\right) n_{i} d S
\end{aligned}
$$

On the other hand, for the r.h.s of Eq. A.2 we have:

$$
\int_{\Gamma} W_{m i} h_{i} d l=\int_{\Gamma} \epsilon_{i n k} w_{m n} b_{k} h_{i} d l=-b_{k} \int_{\Gamma} \epsilon_{k n i} w_{m n} h_{i} d l
$$


Because $b_{k}$ are arbitrary, they can be eliminated from Equations (A.2), (A.5), (A.6); which gives the following second order tensorial equality:

$$
\int_{\Gamma} \epsilon_{k n i} w_{m n} h_{i} d l=\int_{S}\left(\frac{\partial w_{m j}}{\partial x_{j}} \delta_{i k}-\frac{\partial w_{m i}}{\partial x_{k}}\right) n_{i} d S
$$

Now, choosing $\boldsymbol{w}=\vec{x} \otimes \vec{n}$ and knowing that $\epsilon_{k n i} n_{n} h_{i}=\nu_{k}$, Eq. A.7 takes the form:

$$
\int_{\Gamma} \nu_{k} x_{m} d l=\int_{S}\left(n_{m} n_{k}+\frac{\partial n_{j}}{\partial x_{j}} x_{m} n_{k}-\delta_{m k}-x_{m} \frac{\partial n_{i}}{\partial x_{k}} n_{i}\right) d S
$$

Also knowing that:

$$
\frac{\partial n_{i}}{\partial x_{k}} n_{i}=\frac{1}{2} \frac{\partial}{\partial x_{k}}\left(n_{i} n_{i}\right)=\overrightarrow{0}
$$

Equation (A.8) can finally be stated in the form:

$$
\begin{aligned}
\int_{\Gamma} \nu_{k} x_{m} d l & =\int_{S}\left(n_{m} n_{k}+\frac{\partial n_{j}}{\partial x_{j}} x_{m} n_{k}-\delta_{m k}\right) d S \\
\Longleftrightarrow \int_{\Gamma} \vec{\nu} \otimes \vec{x} d l & =\int_{S}\left(\operatorname{div}_{\mathrm{S}}(\vec{n}) \vec{n} \otimes \vec{x}+\vec{n} \otimes \vec{n}-\boldsymbol{\delta}\right) d S
\end{aligned}
$$

which demonstrates Eq. (16).

\section{Appendix B: Demonstration of Eq. (36)}

As a starting point, the trace of the tensorial equality (16) or (A.10) gives for any surface $S$ :

$$
\int_{S}\left(\operatorname{div}_{\mathrm{S}} \vec{n} \vec{n} \cdot \vec{x}-2\right) d S=\int_{\Gamma} \vec{\nu} \cdot \vec{x} d l
$$

Applying Eq. (B.1) to the meniscus lateral surface $S_{m}$ (Fig. 13), we note that $\operatorname{div}_{\mathrm{S}} \vec{n}$ is constant, obeying Laplace-Young equation $\operatorname{div}_{\mathrm{S}} \vec{n}=\left(u_{w}-u_{n}\right) / \gamma_{n w}=$ $-u_{c} / \gamma_{n w}$. Then, the 1.h.s of Eq. (B.1) is:

$$
\int_{S_{m}}\left(\operatorname{div}_{\mathrm{S}} \vec{n} \vec{n} \cdot \vec{x}-2\right) d S=-\frac{u_{c}}{\gamma_{n w}} \int_{S_{m}} \vec{x} \cdot \vec{n} d S-2 S_{m}
$$

The lateral surface $S_{m}$ does not completely enclose the meniscus volume $V_{m}$, however $S_{m} \cup S_{1, s w} \cup S_{2, s w}$ does (Fig. 13). Then, applying the divergence 


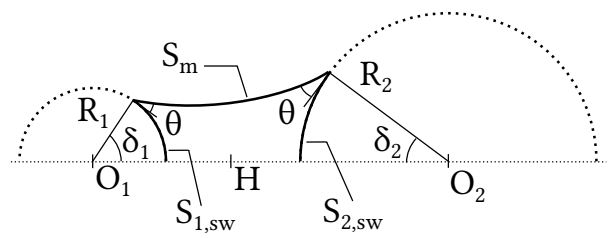

Figure 13: Meniscus half-geometry

theorem with the classical divergence operator div gives:

$$
\begin{aligned}
& -\frac{u_{c}}{\gamma_{n w}} \int_{S_{m}} \vec{x} \cdot \vec{n} d S \\
& =-\frac{u_{c}}{\gamma_{n w}}\left(\int_{V_{m}} \operatorname{div} \vec{x} d V-\sum_{i=1,2} \int_{S_{i, s w}} \vec{x} \cdot \vec{n} d S\right) ; \vec{n} \text { inwards to the particles } \\
& =-\frac{u_{c}}{\gamma_{n w}}\left(3 V_{m}+\sum_{i=1,2} \int_{S_{i, s w}} \vec{x} \cdot \vec{n} d S\right) ; \vec{n} \text { outwards to the particles }
\end{aligned}
$$

For each wetted surface $S_{i, s w}(i=1,2)$ :

$$
\int_{S_{i, s w}} \vec{x} \cdot \vec{n} d S=\int_{S_{i, s w}}\left(\overrightarrow{H O_{i}} \cdot \vec{n}+R_{i} \vec{n} \cdot \vec{n}\right) d S=-H O_{i} \pi R_{i}{ }^{2} \sin ^{2} \delta_{i}+R_{i} S_{i, s w}
$$

The 1.h.s of Eq. (B.1) is finally equal to:

$$
-\frac{u_{c}}{\gamma_{n w}}\left(3 V_{m}+\sum_{i=1,2}\left(R_{i} S_{i, s w}-H O_{i} \pi R_{i}{ }^{2} \sin ^{2} \delta_{i}\right)\right)-2 S_{m}
$$

Focusing now on the r.h.s of Eq. (B.1), we have:

$$
\int_{\Gamma} \vec{\nu} \cdot \vec{x} d l=\int_{\Gamma_{1}} \vec{\nu} \cdot \vec{x} d l+\int_{\Gamma_{2}} \vec{\nu} \cdot \vec{x} d l
$$

For each wetted contour $\Gamma_{i}(i=1,2)$ :

$$
\int_{\Gamma_{i}} \vec{\nu} \cdot \vec{x} d l=\int_{\Gamma_{i}} \vec{\nu} \cdot\left(\overrightarrow{H O_{i}}+R_{i} \vec{n}\right) d l=\Gamma_{i}\left(-H O_{i} \sin \left(\delta_{i}+\theta\right)+R_{i} \sin \theta\right)
$$

Finally, the r.h.s of Eq. (B.1) is:

$$
\int_{\Gamma} \vec{\nu} \cdot \vec{x} d l=\sum_{i=1,2} \Gamma_{i}\left(-H O_{i} \sin \left(\delta_{i}+\theta\right)+R_{i} \sin \theta\right)
$$


Equating (B.5) and (B.8) according to (B.1) gives:

$$
\begin{aligned}
& s\left(3 V_{m}+\sum_{i=1,2} R_{i} S_{i, s w}\right)+\gamma_{n w}\left(2 S_{m}+\sum_{i=1,2} \Gamma_{i} R_{i} \sin \theta\right) \\
& =\sum_{i=1,2} s \pi R_{i}{ }^{2} \sin ^{2} \delta_{i} H O_{i}+\gamma_{n w} \Gamma_{i} H O_{i} \sin \left(\delta_{i}+\theta\right)
\end{aligned}
$$

Since $s \pi R_{1}^{2} \sin ^{2} \delta_{1}+\gamma_{n w} \Gamma_{1} \sin \left(\delta_{1}+\theta\right)=s \pi R_{2}^{2} \sin ^{2} \delta_{2}+\gamma_{n w} \Gamma_{2} \sin \left(\delta_{2}+\theta\right)$ from Eq. (30), the r.h.s of (B.9) is equal to:

$$
\left(s \pi R_{1}^{2} \sin ^{2} \delta_{1}+\gamma_{n w} \Gamma_{1} \sin \left(\delta_{1}+\theta\right)\right) \sum_{i=1,2} H O_{i}
$$

Considering finally that $\sum H O_{i}=R_{1}+R_{2}+d$, this readily demonstrates Eq. (36).

\section{Acknowledgements}

Fruitful discussions with Rakulan Sivanesapillai (Institute of Mechanics, Ruhr-Universität Bochum, Germany) and Dr. Mehdi Pouragha (University of Calgary) are gratefully acknowledged. This work has been funded by the Natural Science and Engineering Research Council of Canada and Foundation Computer Modelling Group.

Alonso, E., Pereira, J.-M., Vaunat, J., Olivella, S., 2010. A microstructurally based effective stress for unsaturated soils. Géotechnique 60, 913-925(12).

Alonso, E. E., Gens, A., Josa, A., 1990. A constitutive model for partially saturated soils. Géotechnique 40 (3), 405-430.

Biot, M. A., 1941. General theory of three-dimensional consolidation. Journal of Applied Physics 12 (2), 155-164.

Bishop, A. W., 1959. The principle of effective stress. Teknisk Ukeblad 106, 859-863. 
Bishop, A. W., Blight, G. E., 1963. Some aspects of effective stress in saturated and partly saturated soils. Géotechnique 13, 177-197.

Borja, R. I., Koliji, A., 2009. On the effective stress in unsaturated porous continua with double porosity. Journal of the Mechanics and Physics of Solids $57(8), 1182-1193$.

Bruchon, J.-F., Pereira, J.-M., Vandamme, M., Lenoir, N., Delage, P., 2013. X-ray microtomography characterisation of the changes in statistical homogeneity of an unsaturated sand during imbibition. Géotechnique Letters 3 (2), $84-88$.

Chateau, X., Moucheront, P., Pitois, O., 2002. Micromechanics of unsaturated granular media. Journal of Engineering Mechanics 128 (8), 856-863.

Culligan, K. A., Wildenschild, D., Christensen, B. S. B., Gray, W. G., Rivers, M. L., Tompson, A. F. B., 2004. Interfacial area measurements for unsaturated flow through a porous medium. Water Resources Research 40 (12).

Cundall, P., Strack, O., 1979. A discrete numerical model for granular assemblies. Géotechnique 29, 47-65.

Dormieux, L., Kondo, D., Ulm, F.-J., 2006a. Microporomechanics. John Wiley \& Sons.

Dormieux, L., Sanahuja, J., Maghous, S., 2006b. Influence of capillary effects on strength of non-saturated porous media. Comptes Rendus Mécanique 334 (1), $19-24$.

Duriez, J., Wan, R., 2016a. Contact angle mechanical influence for wet granular soils. Acta Geotechnica(in press).

Duriez, J., Wan, R., 2016b. Stress in wet granular media with interfaces via homogenization and discrete element approaches. Journal of Engineering Mechanics. 
Duriez, J., Wan, R., 2016c. Subtleties in discrete element modelling of wet granular soils. Géotechnique(in press).

Favier, L., Daudon, D., Donzé, F.-V., Mazars, J., 2009. Predicting the drag coefficient of a granular flow using the discrete element method. Journal of Statistical Mechanics: Theory and Experiment 2009 (06), P06012.

Frederick, C., Armstrong, P., 2007. A mathematical representation of the multiaxial Bauschinger effect. Materials at High Temperatures 24 (1), 1-26.

Fredlund, D., 2006. Unsaturated soil mechanics in engineering practice. Journal of Geotechnical and Geoenvironmental Engineering 132 (3), 286-321.

Gili, J. A., Alonso, E. E., 2002. Microstructural deformation mechanisms of unsaturated granular soils. International Journal for Numerical and Analytical Methods in Geomechanics 26 (5), 433-468.

Gray, W. G., Schrefler, B. A., Pesavento, F., 2009. The solid phase stress tensor in porous media mechanics and the Hill-Mandel condition. Journal of the Mechanics and Physics of Solids 57 (3), 539 - 554.

Gurtin, M. E., Murdoch, A. I., 1975. A continuum theory of elastic material surfaces. Archive for Rational Mechanics and Analysis 57 (4), 291-323.

Hanes, D. M., Walton, O. R., 2000. Simulations and physical measurements of glass spheres flowing down a bumpy incline. Powder Technology 109 (13), 133 -144 .

Hassanizadeh, S., Gray, W. G., 1990. Mechanics and thermodynamics of multiphase flow in porous media including interphase boundaries. Advances in Water Resources 13 (4), 169 - 186.

Henann, D. L., Anand, L., 2009. A large deformation theory for rate-dependent elastic-plastic materials with combined isotropic and kinematic hardening. International Journal of Plasticity 25 (10), 1833 - 1878. 
Herminghaus, S., 2005. Dynamics of wet granular matter. Advances in Physics $54(3), 221-261$.

Hicher, P.-Y., Chang, C., 2007. A microstructural elastoplastic model for unsaturated granular materials. International Journal of Solids and Structures 44 (78), $2304-2323$.

Jiang, Q., Liang, L. H., Zhao, D. S., 2001. Lattice contraction and surface stress of fcc nanocrystals. The Journal of Physical Chemistry B 105 (27), 6275-6277.

Khalili, N., Geiser, F., Blight, G., 2004. Effective stress in unsaturated soils: Review with new evidence. International Journal of Geomechanics 4 (2), 115126.

Lemaitre, J., Chaboche, J.-L., 1990. Mechanics of solid materials. Cambridge university press.

Li, X., Du, Y., Zhang, S., Duan, Q., Schrefler, B., 2016. Meso-hydromechanically informed effective stresses and effective pressures for saturated and unsaturated porous media. European Journal of Mechanics - A/Solids $59,24-36$.

Lu, N., Godt, J. W., Wu, D. T., 2010. A closed-form equation for effective stress in unsaturated soil. Water Resour. Res. 46 (5), 1-14, w05515.

Lu, N., Likos, W., 2006. Suction stress characteristic curve for unsaturated soil. Journal of Geotechnical and Geoenvironmental Engineering 132 (2), 131-142.

Lunati, I., 2007. Young's law and the effects of interfacial energy on the pressure at the solid-fluid interface. Physics of Fluids 19 (11).

Madeo, A., dell'Isola, F., Darve, F., 2013. A continuum model for deformable, second gradient porous media partially saturated with compressible fluids. Journal of the Mechanics and Physics of Solids 61 (11), $2196-2211$. 
Manahiloh, K. N., Muhunthan, B., 2012. Characterizing liquid phase fabric of unsaturated specimens from X-Ray computed tomography images. In: Mancuso, C., Jommi, C., D’Onza, F. (Eds.), Unsaturated Soils: Research and Applications: Volume 1. Springer, pp. 71-80.

Mani, R., Semprebon, C., Kadau, D., Herrmann, H. J., Brinkmann, M., Herminghaus, S., 2015. Role of contact-angle hysteresis for fluid transport in wet granular matter. Phys. Rev. E 91, 042204.

Nikooee, E., Habibagahi, G., Hassanizadeh, S., Ghahramani, A., 2013. Effective stress in unsaturated soils: A thermodynamic approach based on the interfacial energy and hydromechanical coupling. Transport in Porous Media 96 (2), 369-396.

Nowick, A. S., Machlin, E. S., 1947. Dislocation theory as applied by N.A.C.A. to the creep of metals. Journal of Applied Physics 18 (1), 79-87.

Nuth, M., Laloui, L., 2008. Effective stress concept in unsaturated soils: Clarification and validation of a unified framework. International Journal for $\mathrm{Nu}-$ merical and Analytical Methods in Geomechanics 32 (7), 771-801.

Prager, W., 1956. A new method of analysing stresses and strains in workhardening plastic solids. Journal of Applied Mechanics 23 (4), 493-496.

Richefeu, V., Radjaï, F., El Youssoufi, M., 2006. Stress transmission in wet granular materials. The European Physical Journal E 21 (4), 359-369.

Rosenkilde, C. E., 1967. Surface-energy tensors. Journal of Mathematical Physics 8 (1), 84-88.

Scheel, M., Seemann, R., Brinkmann, M., Di Michiel, M., Sheppard, A., Breidenbach, B., Herminghaus, S., 2008. Morphological clues to wet granular pile stability. Nat. Mater. 7 (3), 189-193.

Scholtès, L., Chareyre, B., Nicot, F., Darve, F., 2009. Micromechanics of granular materials with capillary effects. International Journal of Engineering Science $47(1), 64-75$. 
Shikhmurzaev, Y. D., 2008. On Young's (1805) equation and Finn's (2006) 'counterexample'. Physics Letters A 372 (5), 704-707.

Sibille, L., Hadda, N., Nicot, F., Tordesillas, A., Darve, F., 2015. Granular plasticity, a contribution from discrete mechanics. Journal of the Mechanics and Physics of Solids 75, $119-139$.

Sivanesapillai, R., Falkner, N., Hartmaier, A., Steeb, H., 2015. A CSF-SPH method for simulating drainage and imbibition at pore-scale resolution while tracking interfacial areas. Advances in Water Resources, -.

Terzaghi, K., 1936. The shearing resistance of saturated soils and the angle between the planes of shear. In: Proceedings of the 1st International conference on soil mechanics and foundation engineering. Vol. 1. Cambridge, pp. 54-56.

Vermaak, J., Mays, C., Kuhlmann-Wilsdorf, D., 1968. On surface stress and surface tension: I. theoretical considerations. Surface Science 12 (2), 128 133.

Vitz, E., 1990. Magic sand: Modeling the hydrophobic effect and reversed-phase liquid chromatography. Journal of Chemical Education 67 (6), 512.

Šmilauer, V., et al., 2015. Yade Documentation 2nd ed. The Yade Project, http://yade-dem.org/doc/.

Wan, R., Duriez, J., Darve, F., 2015. A tensorial description of stresses in triphasic granular materials with interfaces. Geomechanics for Energy and the Environment 4, $73-87$.

Wang, K., Sun, W., 2015. Anisotropy of a tensorial Bishop's coefficient for wetted granular materials. Journal of Engineering Mechanics 0 (0), B4015004.

Young, T., 1805. An essay on the cohesion of fluids. Philosophical Transactions of the Royal Society of London 95, 65-87. 
Zienkiewicz, O. C., Chan, A. H. C., Pastor, M., Schrefler, B. A., Shiomi, T., 1999. Computational geomechanics with special reference to earthquake engineering. Wiley. 